\title{
Localization effects in a periodic quantum graph with magnetic field and spin-orbit interaction
}

\author{
KONSTANTIN PANKRASHKIN \\ Institut für Mathematik, Humboldt-Universität zu Berlin, Rudower Chaussee 25, \\ 12489 Berlin, Germany \\ E-mail: constemathematik.hu-berlin.de \\ Homepage: http: //www.mathematik.hu-berlin.de/ const/
}

\begin{abstract}
A general technique for the study of magnetic Rashba Hamiltonian in quantum graphs is presented. We use this technique to show how manipulating the magnetic and spin parameters can be used to create localized states in a certain periodic graph ( $T_{3}$ lattice).
\end{abstract}

\section{Introduction}

In this work, we discuss the creation of eigenvalues in periodic quantum graphs by certain external interactions, namely, by magnetic field and spin-orbit coupling.

The analysis of quantum graphs, i.e. of differential operators on singular one-dimensional manifolds, becomes one the central topics in the mathematical physics during last decades, see $[28,29,31-33,35]$. This has many reasons; in particular, quantum graph Hamiltonians appear in the de Gennes-Alexander theory of superconductivity $[4,15,25]$. Some other fields of applications are described e.g. in [31].

The spectral theory of compact quantum graphs has many common features with the usual theory of differential operators, cf. [6,42,48]. Nevertheless, such an analogy is rather limited when considering non-compact structures. Some particular features of quantum graph models become obvious if one studies periodic configurations. For example, for a large class of periodic Schrödinger operators in Euclidian spaces the spectrum is known to be absolutely continuous [9,50], while even simplest periodic quantum graphs can have eigenvalues [12,33]. Some other examples may include the sensibility of periodic quantum graphs to some arithmetic characteristics [24].

Recently, in the physics literature one discussed the so-called extreme localization in the $T_{3}$ lattice (dice lattice) $[1,54]$. From the mathematical point of view, it was shown that under certain magnetic fields the Hamiltonian of a quantum graph with the corresponding shape has no bands of continuous spectrum, and 
the spectrum consists of infinitely degenerate eigenvalues. This effect was observed also experimentally by transport measuring in superconducting and metallic wire networks [39-41]. (It is worth emphasizing that bound states in $T_{3}$ lattice appear without any external interactions [51]; the coexistence of the continuous and the point spectra is implied by the rich internal symmetry of the lattice and of its dual, Kagomé [2].) Various aspects of this localization mechanism and its stability under disorder and external interactions are studied in a number of works $[8,38,52-54]$. In particular, it is shown that additional interactions, like the inter-particle interaction, destroy the extreme localization mechanism, and continuous spectrum appears [53].

In Ref. [7] it was shown that in some periodic quantum graphs similar localization phenomena can be induced not only by magnetic fields, but also by spin-orbit interaction at certain values of the Rashba constant [14,47]. Nevertheless, this analogy is limited, and the numerical analysis of [8] shows that the Rashba localization does not appear in the $T_{3}$ lattice. In the present paper we consider the above situation with both the spin-orbit and magnetic interactions. We note that the quantum graph models with spin were studied previously e.g. in $[10,11,13,27]$, but the attention was mostly concentrated on Dirac- and Paulitype operators. The theory of Rashba Hamiltonians is not developed even in the Euclidian spaces, where the spin-orbit interaction promises to show effects which are absent in the scalar case, like embedded eigenvalues in short-range potentials or localization in crystals, giving hence possibilities for constructing new nanodevices $[17,34]$.

The aim of the paper is two-fold. First, we are going to describe the Schrödinger operators in two-dimensional networks with magnetic field and spinorbit interaction. An essential part here is the reduction of the quantum graph Hamiltonian to a certain discrete equation. In the scalar case, an analogous procedure was done in [23] for the solutions of the stationary Schrödinger equation and recently in [44] for the spectra. Note that there is another approach to the relationship between the quantum graphs and tight-binding Hamiltonians coming from some asymptotic considerations $[36,37]$. Second, by considering the localization effects in the $T_{3}$-lattice we would like to attract the attention of researchers working on quantum graphs to potential applications in the study of superconducting networks. In section 2 we give a mathematical formalism of quantum graphs with external interactions; essentially we describe rigorously the constructions of the works $[7,8]$. In section 3 we use this machinery to study the spectrum of the $T_{3}$ lattice with a magnetic field and the Rashba interaction. We show that the spectral problem is of supersymmetric type and that the study of some energy levels is equivalent to the study of zero modes in a certain discrete model. As a result, we give a rigorous justification of the extreme localization for the case of non-trivial scalar potentials on the edges and non-ideal couplings at the nodes. We show that 
at zero spin-orbit interaction this effect is independent of the edge potential. At the same time, it appears that the generic Rashba interaction destroys the localization. We also show that at a certain combination of the magnetic and spin parameters a new localization effect appears, where one can localize one of the spin projections using the magnetic field.

\section{Quantum graphs with external interactions}

\subsection{Schrödinger operator on a quantum graph embedded in Euclidian space}

In this section we describe the construction of the Hamiltonian in a twodimensional network with magnetic field and Rashba interaction. Recall that the Rashba Hamiltonian of a two-dimensional system acts on two-component vector functions as takes the form $[14,47]$

$$
H=(\mathbf{p}-\mathbf{A})^{2}+2 k_{R}\langle\mathbf{p}-\mathbf{A}, \boldsymbol{\sigma} \times \mathbf{n}\rangle+U,
$$

where $\mathbf{A}$ is the magnetic vector potential, $k_{R}$ is the Rashba constant expressing the strength of the spin-orbit interaction, $U$ is a scalar potential, $\sigma$ is the vector of Pauli matrices, and $\mathbf{n}$ is the unit vector orthogonal to the plane of the system. The second term, which is the formal mixed product, on the right-hand side of (1) takes into account the spin-orbit coupling. For $k_{R}=0$ the problem splits in two identical scalar problems. The corresponding Hamiltonian for a network is obtained by projecting all the interactions onto each edge and by introducing suitable boundary conditions at the nodes, which will be described below. (We remark that some effects of the Rashba interaction and the magnetic field in a wire can be studied in other types of models [19].)

Let $\mathscr{V}$ be a uniformly discrete subset of the $x y$-plane in $\mathbb{R}^{3}$, the set of nodes (vertices). The uniform discreteness means the existence of a constant $d>0$ such that $|\boldsymbol{\alpha}-\boldsymbol{\beta}| \geq d$ for all $\boldsymbol{\alpha}, \boldsymbol{\beta} \in \mathscr{V}$ with $\boldsymbol{\alpha} \neq \boldsymbol{\beta}$. Denote

$$
l_{\boldsymbol{\alpha} \beta}:=|\boldsymbol{\alpha}-\boldsymbol{\beta}|, \quad \mathbf{e}_{\boldsymbol{\alpha} \boldsymbol{\beta}}:=\frac{1}{l_{\boldsymbol{\alpha} \boldsymbol{\beta}}}(\boldsymbol{\beta}-\boldsymbol{\alpha}) .
$$

Some nodes are connected by a directed edge. The set of all edges will be denoted by $\mathscr{E}, \mathscr{E} \subset \mathscr{V} \times \mathscr{V}$. The edge with initial vertex $\boldsymbol{\alpha} \in \mathscr{V}$ and terminal vertex $\boldsymbol{\beta} \in \mathscr{V}$ will be denoted by $\boldsymbol{\alpha} \boldsymbol{\beta}$. For $\boldsymbol{\alpha} \in \mathscr{V}$ denote indeg $\boldsymbol{\alpha}:=\#\{\boldsymbol{\beta} \boldsymbol{\alpha} \in \mathscr{E}\}$, outdeg $\boldsymbol{\alpha}=$ $\#\{\boldsymbol{\alpha} \boldsymbol{\beta} \in \mathscr{E}\}, \operatorname{deg} \boldsymbol{\alpha}:=\operatorname{indeg} \boldsymbol{\alpha}+\operatorname{outdeg} \boldsymbol{\alpha}$. We assume that the degrees satisfy the following conditions: that

$$
\text { there exists } N \in \mathbb{Z} \text { with } 1 \leq \operatorname{deg} \boldsymbol{\alpha} \leq N \text { for all } \boldsymbol{\alpha} \in \mathscr{V}
$$


in particular, we assume that there are no isolated vertices. The configuration consisting of all segments $[\boldsymbol{\alpha}, \boldsymbol{\beta}], \boldsymbol{\alpha} \boldsymbol{\beta} \in \mathscr{E}$ will be referred to as a metric graph or as a (wire) network. We assume that the system has no self-intersections and that

$$
0<\inf \left\{l_{\boldsymbol{\alpha} \boldsymbol{\beta}}\right\} \leq \sup \left\{l_{\boldsymbol{\alpha} \boldsymbol{\beta}}\right\}<\infty
$$

The quantum state space corresponding to the metric graph is introduced as follows. Each edge $\boldsymbol{\alpha} \boldsymbol{\beta}$ will be identified with the segment $\left[0, l_{\boldsymbol{\alpha} \boldsymbol{\beta}}\right]$ such that $\boldsymbol{\alpha}$ is identified with 0 and $\boldsymbol{\beta}$ is identified with $l_{\boldsymbol{\alpha} \boldsymbol{\beta}}$. The state space of each edge $\boldsymbol{\alpha} \boldsymbol{\beta}$ is $\mathscr{H}_{\boldsymbol{\alpha} \boldsymbol{\beta}}:=L^{2}\left(\left[0, l_{\boldsymbol{\alpha} \boldsymbol{\beta}}\right], \mathbb{C}^{2}\right)$. The state space of the whole structure is $\mathscr{H}=$ $\bigoplus_{\boldsymbol{\alpha} \boldsymbol{\beta} \in \mathscr{E}} \mathscr{H}_{\boldsymbol{\alpha} \boldsymbol{\beta}}$.

On each edge consider a real-valued scalar potential $U_{\boldsymbol{\alpha} \boldsymbol{\beta}} \in L^{2}\left[0, l_{\boldsymbol{\alpha} \boldsymbol{\beta}}\right]$. To avoid unnecessary technical difficulties we will assume that the scalar potentials are uniformly $L^{2}$-bounded,

$$
\sup \left\|U_{\boldsymbol{\alpha} \boldsymbol{\beta}}\right\|_{L^{2}}<\infty
$$

Assume that the system is subjected to an external magnetic field given by a vector potential $\mathbf{A} \in C^{1}\left(\mathbb{R}^{3}, \mathbb{R}^{3}\right)$. This induces magnetic potentials on each edge, $a_{\boldsymbol{\alpha} \boldsymbol{\beta}}(t):=\left\langle\mathbf{A}\left(\boldsymbol{\alpha}+t \mathbf{e}_{\boldsymbol{\alpha} \boldsymbol{\beta}}\right), \mathbf{e}_{\boldsymbol{\alpha} \boldsymbol{\beta}}\right\rangle$.

Denote by $k_{R}$ the Rashba constant. The spin-orbit interaction can be taken into account by adding the term $2 k_{R}\left(-\mathrm{i} \frac{\mathrm{d}}{\mathrm{d} t}-a_{\boldsymbol{\alpha} \boldsymbol{\beta}}(t)\right)\left\langle\boldsymbol{\sigma} \times \mathbf{n}, \mathbf{e}_{\boldsymbol{\alpha} \boldsymbol{\beta}}\right\rangle$ with $\mathbf{n}=(0,0,1)$. Therefore, the dynamics along each edge $\boldsymbol{\alpha} \boldsymbol{\beta}$ is described by the differential expression

$$
\begin{aligned}
L_{\boldsymbol{\alpha} \boldsymbol{\beta}}=\left(-\mathrm{i} \frac{\mathrm{d}}{\mathrm{d} t}-a_{\boldsymbol{\alpha} \boldsymbol{\beta}}(t)\right)^{2}+2 k_{R}( & \left.-\mathrm{i} \frac{\mathrm{d}}{\mathrm{d} t}-a_{\boldsymbol{\alpha} \boldsymbol{\beta}}(t)\right)\left\langle\boldsymbol{\sigma} \times \mathbf{n}, \mathbf{e}_{\boldsymbol{\alpha} \boldsymbol{\beta}}\right\rangle+U_{\boldsymbol{\alpha} \boldsymbol{\beta}} \\
& \equiv\left(\mathrm{i} \frac{\mathrm{d}}{\mathrm{d} t}+a_{\boldsymbol{\alpha} \boldsymbol{\beta}}(t)+k_{R} \sigma_{\boldsymbol{\alpha} \boldsymbol{\beta}}\right)^{2}+U_{\boldsymbol{\alpha} \boldsymbol{\beta}}-k_{R}^{2},
\end{aligned}
$$

where

$$
\sigma_{\alpha \beta}=\left(\begin{array}{cc}
0 & e_{\alpha \beta 2}+\mathrm{i} e_{\boldsymbol{\alpha} \beta 1} \\
e_{\boldsymbol{\alpha} \beta 2}-\mathrm{i} e_{\boldsymbol{\alpha} \boldsymbol{\beta} 1} & 0
\end{array}\right) .
$$

For a uniform magnetic field with the strength $\mathbf{B} \in \mathbb{R}^{3}$ it is useful to use the symmetric gauge, $\mathbf{A}(\mathbf{r})=\frac{1}{2} \mathbf{B} \times \mathbf{r}$. In this case the magnetic potentials $a_{\boldsymbol{\alpha} \beta}$ are constant, $a_{\boldsymbol{\alpha} \boldsymbol{\beta}}=\frac{1}{2}\left\langle\mathbf{B} \times \boldsymbol{\alpha}, \mathbf{e}_{\boldsymbol{\alpha} \boldsymbol{\beta}}\right\rangle$.

Denote by $L$ an operator in $\mathscr{H}$ acting as

$$
\left(\mathbf{f}_{\boldsymbol{\alpha} \boldsymbol{\beta}}\right) \mapsto\left(L_{\boldsymbol{\alpha} \boldsymbol{\beta}} \mathbf{f}_{\boldsymbol{\alpha} \boldsymbol{\beta}}\right)
$$


on functions $\mathbf{f}_{\boldsymbol{\alpha} \boldsymbol{\beta}} \in H^{2}\left(\left[0, l_{\boldsymbol{\alpha} \boldsymbol{\beta}}\right], \mathbb{C}^{2}\right)$ satisfying at each $\boldsymbol{\alpha} \in \mathscr{V}$ :

$$
\begin{gathered}
\mathbf{f}_{\boldsymbol{\alpha} \boldsymbol{\beta}}(0)=\mathbf{f}_{\boldsymbol{\gamma} \boldsymbol{\alpha}}\left(l_{\boldsymbol{\gamma} \boldsymbol{\alpha}}\right)=: \mathbf{f}(\boldsymbol{\alpha}), \quad \boldsymbol{\alpha} \boldsymbol{\beta}, \boldsymbol{\gamma} \boldsymbol{\alpha} \in \mathscr{E} \\
\sum_{\boldsymbol{\alpha} \boldsymbol{\beta} \in \mathscr{E}}\left[\frac{\mathrm{d}}{\mathrm{d} t}-\mathrm{i}\left(a_{\boldsymbol{\alpha} \boldsymbol{\beta}}+k_{R} \sigma_{\boldsymbol{\alpha} \boldsymbol{\beta}}\right)\right] \mathbf{f}_{\boldsymbol{\alpha} \boldsymbol{\beta}}(0) \\
-\sum_{\boldsymbol{\beta} \boldsymbol{\alpha} \in \mathscr{E}}\left[\frac{\mathrm{d}}{\mathrm{d} t}-\mathrm{i}\left(a_{\boldsymbol{\beta} \boldsymbol{\alpha}}+k_{R} \sigma_{\boldsymbol{\beta} \boldsymbol{\alpha}}\right)\right] \mathbf{f}_{\boldsymbol{\beta} \boldsymbol{\alpha}}\left(l_{\boldsymbol{\beta} \boldsymbol{\alpha}}\right)=\varepsilon(\boldsymbol{\alpha}) \mathbf{f}(\alpha),
\end{gathered}
$$

where $\varepsilon(\boldsymbol{\alpha})$ are real-valued parameters. The case $\varepsilon(\boldsymbol{\alpha})=0$ may be considered as an ideal coupling, which is the analogue of the Kirchhoff coupling in the scalar case. We are going to consider $L$ as the Hamiltonian of the system, and our next aim is to show its self-adjointness.

\subsection{Self-adjointness and spectral analysis}

Denote by $\mathscr{D}$ the set of all functions $\mathbf{f}=\left(\mathbf{f}_{\boldsymbol{\alpha} \beta}\right)$, with components $\left(\mathbf{f}_{\boldsymbol{\alpha} \boldsymbol{\beta}}\right) \in$ $\bigoplus H^{2}\left(\left[0, l_{\boldsymbol{\alpha} \boldsymbol{\beta}}\right], \mathbb{C}^{2}\right), \boldsymbol{\alpha} \boldsymbol{\beta} \in \mathscr{E}$, which are continuous at all nodes, i.e. such that the condition (6a) is satisfied. Clearly, for $\mathbf{f} \in \mathscr{D}$ the values $\mathbf{f}(\boldsymbol{\alpha}), \boldsymbol{\alpha} \in \mathscr{V}$, have the direct sense. Furthermore, for $\mathbf{f} \in \mathscr{D}$ and $\boldsymbol{\alpha} \in \mathscr{V}$ denote

$$
\begin{aligned}
\mathbf{f}^{\prime}(\boldsymbol{\alpha}):=\sum_{\boldsymbol{\alpha} \boldsymbol{\beta} \in \mathscr{E}}\left[\frac{\mathrm{d}}{\mathrm{d} t}-\mathrm{i}\left(a_{\boldsymbol{\alpha} \boldsymbol{\beta}}+k_{R} \sigma_{\boldsymbol{\alpha} \boldsymbol{\beta}}\right)\right] \mathbf{f}_{\boldsymbol{\alpha} \boldsymbol{\beta}}(0) & \\
& -\sum_{\boldsymbol{\beta} \boldsymbol{\alpha} \in \mathscr{E}}\left[\frac{\mathrm{d}}{\mathrm{d} t}-\mathrm{i}\left(a_{\boldsymbol{\beta} \boldsymbol{\alpha}}+k_{R} \sigma_{\boldsymbol{\beta} \boldsymbol{\alpha}}\right)\right] \mathbf{f}_{\boldsymbol{\beta} \boldsymbol{\alpha}}\left(l_{\boldsymbol{\beta} \boldsymbol{\alpha}}\right) .
\end{aligned}
$$

Consider in $\mathscr{H}$ a linear operator $\Pi$ with domain $\mathscr{D}$ acting by the rule (5).

Proposition 1. The operator $\Pi$ is closed. For any $\mathbf{f} \in \operatorname{dom} \Pi \equiv \mathscr{D}$ the vectors $\Gamma \mathbf{f}:=$ $(\mathbf{f}(\boldsymbol{\alpha}))$ and $\Gamma^{\prime} \mathbf{f}:=\left(\mathbf{f}^{\prime}(\boldsymbol{\alpha})\right)$ belong to $\ell^{2}\left(\mathscr{V}, \mathbb{C}^{2}\right)$, and the map $\left(\Gamma, \Gamma^{\prime}\right): \operatorname{dom} L \rightarrow$ $\ell^{2}\left(\mathscr{V}, \mathbb{C}^{2}\right) \oplus \ell^{2}\left(\mathscr{V}, \mathbb{C}^{2}\right)$ is surjective. For any $\mathbf{f}, \mathbf{g} \in \operatorname{dom} \Pi$ there holds

$$
\langle\mathbf{f}, \Pi \mathbf{g}\rangle-\langle\Pi \mathbf{f}, \mathbf{g}\rangle=\left\langle\Gamma \mathbf{f}, \Gamma^{\prime} \mathbf{g}\right\rangle-\left\langle\Gamma^{\prime} \mathbf{f}, \Gamma \mathbf{g}\right\rangle .
$$

Proof. Denote by $\Theta_{\alpha \beta}$ the unitary transformation of $\mathscr{H}_{\boldsymbol{\alpha} \boldsymbol{\beta}}$ given by

$$
\Theta_{\boldsymbol{\alpha} \boldsymbol{\beta}} \mathbf{f}(t)=\exp \left(\mathrm{i} \int_{0}^{t}\left(a_{\boldsymbol{\alpha} \boldsymbol{\beta}}(s)+k_{R} \sigma_{\boldsymbol{\alpha} \boldsymbol{\beta}}\right) \mathrm{d} s\right) \mathbf{f}(t) .
$$

Denoting $\partial:=\frac{\mathrm{d}}{\mathrm{d} t}$ we see $\left(\partial-\mathrm{i} a_{\boldsymbol{\alpha} \beta}-\mathrm{i} k_{R} \sigma_{\alpha \beta}\right) \Theta_{\alpha \beta} \equiv \Theta_{\alpha \beta} \partial$. 
By the Sobolev inequality, for any $c_{1}>0$ there exists $c_{2}>0$ such that for any $l>0$ and $\varphi \in H^{2}[0, l]$ there holds

$$
\begin{aligned}
& \|\varphi\|_{\infty} \leq c_{1} l^{3 / 2}\left\|\varphi^{\prime \prime}\right\|_{L^{2}[0, l]}+\frac{c_{2}}{l^{1 / 2}}\|\varphi\|_{L^{2}[0, l]}, \\
& \left\|\varphi^{\prime}\right\|_{\infty} \leq c_{1} l^{1 / 2}\left\|\varphi^{\prime \prime}\right\|_{L^{2}[0, l]}+\frac{c_{2}}{l^{1 / 2}}\|\varphi\|_{L^{2}[0, l]} .
\end{aligned}
$$

Note that for any $t \in\left[0, l_{\boldsymbol{\alpha} \boldsymbol{\beta}}\right]$ one has $\left\|\mathbf{f}_{\boldsymbol{\alpha} \boldsymbol{\beta}}(t)\right\|_{\mathbb{C}^{2}}=\left\|\Theta_{\boldsymbol{\alpha} \boldsymbol{\beta}} \mathbf{f}_{\boldsymbol{\alpha} \boldsymbol{\beta}}(t)\right\|_{\mathbb{C}^{2}}$. Therefore, using the above estimate, for any $\mathbf{f}_{\boldsymbol{\alpha} \boldsymbol{\beta}} \in H^{2}\left(\left[0, l_{\boldsymbol{\alpha} \boldsymbol{\beta}}\right], \mathbb{C}^{2}\right)$ one has

$$
\begin{aligned}
\left\|\mathbf{f}_{\boldsymbol{\alpha} \boldsymbol{\beta}}(t)\right\|_{\mathbb{C}^{2}} & =\left\|\Theta_{\boldsymbol{\alpha} \boldsymbol{\beta}}^{*} \mathbf{f}_{\boldsymbol{\alpha} \boldsymbol{\beta}}(t)\right\|_{\mathbb{C}^{2}} \\
& \leq c_{1} l_{\boldsymbol{\alpha} \boldsymbol{\beta}}^{3 / 2}\left\|\partial^{2} \Theta_{\boldsymbol{\alpha} \boldsymbol{\beta}}^{*} \mathbf{f}_{\boldsymbol{\alpha} \boldsymbol{\beta}}\right\|_{\mathscr{H}_{\boldsymbol{\alpha} \boldsymbol{\beta}}}+\frac{c_{2}}{l_{\boldsymbol{\alpha} \boldsymbol{\beta}}^{1 / 2}}\left\|\Theta_{\boldsymbol{\alpha} \boldsymbol{\beta}}^{*} \mathbf{f}_{\boldsymbol{\alpha} \boldsymbol{\beta}}\right\|_{\mathscr{H}_{\boldsymbol{\alpha} \boldsymbol{\beta}}} \\
& =c_{1} l_{\boldsymbol{\alpha} \boldsymbol{\beta}}^{3 / 2}\left\|\Theta_{\boldsymbol{\alpha} \boldsymbol{\beta}}^{*}\left(\partial-\mathrm{i} a_{\boldsymbol{\alpha} \boldsymbol{\beta}}-\mathrm{i} k_{R} \sigma_{\boldsymbol{\alpha} \boldsymbol{\beta}}\right)^{2} \mathbf{f}_{\boldsymbol{\alpha} \boldsymbol{\beta}}\right\|_{\mathscr{H}_{\boldsymbol{\alpha} \boldsymbol{\beta}}}+\frac{c_{2}}{l_{\boldsymbol{\alpha} \boldsymbol{\beta}}^{1 / 2}}\left\|\Theta_{\boldsymbol{\alpha} \boldsymbol{\beta}}^{*} \mathbf{f}_{\boldsymbol{\alpha} \boldsymbol{\beta}}\right\|_{\mathscr{H}_{\boldsymbol{\alpha} \boldsymbol{\beta}}} \\
& =c_{1} l_{\boldsymbol{\alpha} \boldsymbol{\beta}}^{3 / 2}\left\|\left(\partial-\mathrm{i} a_{\boldsymbol{\alpha} \boldsymbol{\beta}}-\mathrm{i} k_{R} \sigma_{\boldsymbol{\alpha} \boldsymbol{\beta}}\right)^{2} \mathbf{f}_{\boldsymbol{\alpha} \boldsymbol{\beta}}\right\|_{\mathscr{H}_{\boldsymbol{\alpha} \boldsymbol{\beta}}}+\frac{c_{2}}{l_{\boldsymbol{\alpha} \boldsymbol{\beta}}^{1 / 2}}\left\|\mathbf{f}_{\boldsymbol{\alpha} \boldsymbol{\beta}}\right\|_{\mathscr{H}_{\boldsymbol{\alpha} \boldsymbol{\beta}}}
\end{aligned}
$$

and, in the same way,

$$
\begin{aligned}
\left\|\left(\partial-\mathrm{i} a_{\boldsymbol{\alpha} \boldsymbol{\beta}}-\mathrm{i} k_{R} \sigma_{\boldsymbol{\alpha} \boldsymbol{\beta}}\right) \mathbf{f}_{\boldsymbol{\alpha} \boldsymbol{\beta}}(t)\right\|_{\mathbb{C}^{2}} & \\
& \leq c_{1} l_{\boldsymbol{\alpha} \boldsymbol{\beta}}^{1 / 2}\left\|\left(\partial-\mathrm{i} a_{\boldsymbol{\alpha} \boldsymbol{\beta}}-\mathrm{i} k_{R} \sigma_{\boldsymbol{\alpha} \boldsymbol{\beta}}\right)^{2} \mathbf{f}_{\boldsymbol{\alpha} \boldsymbol{\beta}}\right\|_{\mathscr{H}_{\boldsymbol{\alpha} \boldsymbol{\beta}}}+\frac{c_{2}}{l_{\boldsymbol{\alpha} \boldsymbol{\beta}}^{1 / 2}}\left\|\mathbf{f}_{\boldsymbol{\alpha} \boldsymbol{\beta}}\right\|_{\mathscr{H}_{\boldsymbol{\alpha} \boldsymbol{\beta}}} .
\end{aligned}
$$

Using the assumptions (3) and (4) we conclude that there exist positive constants $C_{1}$ and $C_{2}$ such that for any $\boldsymbol{\alpha} \boldsymbol{\beta} \in \mathscr{E}, \mathbf{f}_{\boldsymbol{\alpha} \boldsymbol{\beta}} \in H^{2}\left(\left[0, l_{\boldsymbol{\alpha} \boldsymbol{\beta}}\right], \mathbb{C}^{2}\right), t \in\left[0, l_{\boldsymbol{\alpha} \boldsymbol{\beta}}\right]$ one has

$$
\begin{gathered}
\left\|\mathbf{f}_{\boldsymbol{\alpha} \boldsymbol{\beta}}(t)\right\| \leq C_{1}\left\|L_{\boldsymbol{\alpha} \boldsymbol{\beta}} f_{\boldsymbol{\alpha} \boldsymbol{\beta}}\right\|+C_{2}\left\|f_{\boldsymbol{\alpha} \boldsymbol{\beta}}\right\|, \\
\left\|\left(\partial-\mathrm{i} a_{\boldsymbol{\alpha} \boldsymbol{\beta}}-\mathrm{i} k_{R} \sigma_{\boldsymbol{\alpha} \boldsymbol{\beta}}\right) \mathbf{f}_{\boldsymbol{\alpha} \boldsymbol{\beta}}(t)\right\| \leq C_{1}\left\|L_{\boldsymbol{\alpha} \boldsymbol{\beta}} f_{\boldsymbol{\alpha} \boldsymbol{\beta}}\right\|+C_{2}\left\|f_{\boldsymbol{\alpha} \boldsymbol{\beta}}\right\| .
\end{gathered}
$$

Here the norms are taken in $\mathbb{C}^{2}$ on the left-hand side and in $\mathscr{H}_{\boldsymbol{\alpha} \boldsymbol{\beta}}$ on the right-hand side.

Denote by $\widetilde{\Pi}$ the operator acting in $\mathscr{H}$ by the rule (5) on the domain dom $\widetilde{\Pi}=$ $\bigoplus_{\boldsymbol{\alpha} \boldsymbol{\beta} \in \mathscr{E}} H^{2}\left(\left[0, l_{\boldsymbol{\alpha} \boldsymbol{\beta}}\right], \mathbb{C}^{2}\right)$. Clearly, $\widetilde{\Pi}$ is closed. By (9a), the linear maps

$$
T_{\boldsymbol{\alpha} \boldsymbol{\beta} \gamma}: \operatorname{dom} \widetilde{\Pi} \ni \mathbf{f} \mapsto \mathbf{f}_{\boldsymbol{\alpha} \boldsymbol{\beta}}(0)-\mathbf{f}_{\boldsymbol{\gamma} \boldsymbol{\alpha}}\left(l_{\boldsymbol{\gamma} \boldsymbol{\alpha}}\right) \in \mathbb{C}^{2}, \quad \boldsymbol{\alpha} \boldsymbol{\beta}, \boldsymbol{\gamma} \boldsymbol{\alpha} \in \mathscr{E}
$$

are bounded with respect to the graph norm of $\widetilde{\Pi}$. Therefore, the restriction of $\widetilde{\Pi}$ to the subspace where all these functionals vanish is a closed operator. As this restriction is exactly $\Pi$, the operator $\Pi$ is closed. 
For $\mathbf{f} \in \mathscr{D}$ the inclusions $\Gamma \mathbf{f}, \Gamma^{\prime} \mathbf{f} \in \ell^{2}\left(\mathscr{V}, \mathbb{C}^{2}\right)$ follow immediately from the estimates (9) and the assumption (2), and the identity (7) can be verified directly using the partial integration.

To prove the surjectivity condition, we fix first four functions $f_{j k} \in H^{2}[0,1]$ with $f_{j k}^{(i)}(l)=\delta_{i j} \delta_{k l}, i, j, k, l \in\{0,1\}$. Take arbitrary $\boldsymbol{\xi}, \boldsymbol{\xi}^{\prime} \in \ell^{2}\left(\mathscr{V}, \mathbb{C}^{2}\right)$. Denote

$$
\begin{aligned}
\tau_{\boldsymbol{\alpha} \boldsymbol{\beta}}:= & \exp \left(\mathrm{i} \int_{0}^{l_{\boldsymbol{\alpha} \boldsymbol{\beta}}}\left(a_{\boldsymbol{\alpha} \boldsymbol{\beta}}(s)+k_{R} \sigma_{\boldsymbol{\alpha} \boldsymbol{\beta}}\right) \mathrm{d} s\right) \\
& \equiv \exp \left(\mathrm{i} \int_{0}^{l_{\boldsymbol{\alpha} \boldsymbol{\beta}}} a_{\boldsymbol{\alpha} \boldsymbol{\beta}}(s) \mathrm{d} s\right)\left(\cos k_{R} l_{\boldsymbol{\alpha} \boldsymbol{\beta}}+\mathrm{i} \sigma_{\boldsymbol{\alpha} \boldsymbol{\beta}} \sin k_{R} l_{\boldsymbol{\alpha} \boldsymbol{\beta}}\right) \in \mathbf{U}(2) .
\end{aligned}
$$

By direct calculation, the function $\mathbf{f} \in \mathscr{H}$ whose components are of the form $\mathbf{f}_{\alpha \beta}=\Theta_{\alpha \beta} \mathbf{g}_{\alpha \beta}$, where

$$
\begin{aligned}
\mathbf{g}_{\boldsymbol{\alpha} \boldsymbol{\beta}}(t)=f_{00}\left(\frac{t}{l_{\boldsymbol{\alpha} \boldsymbol{\beta}}}\right) \boldsymbol{\xi} & (\boldsymbol{\alpha})+f_{01}\left(\frac{t}{l_{\boldsymbol{\alpha} \boldsymbol{\beta}}}\right) \tau_{\boldsymbol{\alpha} \boldsymbol{\beta}}^{*} \boldsymbol{\xi}(\boldsymbol{\beta}) \\
& +\frac{l_{\boldsymbol{\alpha} \boldsymbol{\beta}}}{\operatorname{deg} \boldsymbol{\alpha}} f_{10}\left(\frac{t}{l_{\boldsymbol{\alpha} \boldsymbol{\beta}}}\right) \boldsymbol{\xi}^{\prime}(\boldsymbol{\alpha})-\frac{l_{\boldsymbol{\alpha} \boldsymbol{\beta}}}{\operatorname{deg} \boldsymbol{\beta}} f_{11}\left(\frac{t}{l_{\boldsymbol{\alpha} \boldsymbol{\beta}}}\right) \tau_{\boldsymbol{\alpha} \boldsymbol{\beta}}^{*} \boldsymbol{\xi}^{\prime}(\boldsymbol{\beta}),
\end{aligned}
$$

lies in $\mathscr{D}$ and satisfies $\left(\Gamma \mathbf{f}, \Gamma^{\prime} \mathbf{f}\right)=\left(\boldsymbol{\xi}, \boldsymbol{\xi}^{\prime}\right)$.

Proposition 1 shows that the space $\mathscr{G}:=\ell^{2}\left(\mathscr{V}, \mathbb{C}^{2}\right)$ and the maps $\Gamma, \Gamma^{\prime}$ : $\operatorname{dom} \Pi \rightarrow \mathscr{G}$ form a boundary triple for $\Pi$, see e.g. $[21,46]$ for a detailed discussion. The self-adjointness of $\Pi$ would follow from the following assertion [21]: if $\Pi$ has at least one self-adjoint restriction (i.e. if $\Pi^{*}$ is symmetric) and $A$ is a selfadjoint operator in $\mathscr{G}$, then the restriction of $\Pi$ to the vectors $\varphi \in \operatorname{dom} \Pi$ satisfying $\Gamma^{\prime} \varphi=A \Gamma \varphi$ is self-adjoint in $\mathscr{H}$.

Consider the restriction $D$ of $\Pi$ to the functions $\mathbf{f}$ satisfying $\Gamma \mathbf{f}=\mathbf{0}$. Clearly, this restriction is nothing but the direct sum $\bigoplus_{\alpha \beta \in \mathscr{E}} D_{\alpha \beta}$, where $D_{\alpha \beta}$ is an operator in $\mathscr{H}_{\boldsymbol{\alpha} \boldsymbol{\beta}}$ acting as $\mathbf{f}_{\boldsymbol{\alpha} \boldsymbol{\beta}} \mapsto L_{\boldsymbol{\alpha} \boldsymbol{\beta}} \mathbf{f}_{\boldsymbol{\alpha} \boldsymbol{\beta}}$ on functions satisfying $\mathbf{f}_{\boldsymbol{\alpha} \boldsymbol{\beta}}(0)=\mathbf{f}_{\boldsymbol{\alpha} \boldsymbol{\beta}}\left(l_{\boldsymbol{\alpha} \boldsymbol{\beta}}\right)=$ 0. As each $D_{\alpha \beta}$ is self-adjoint, so is $D$. Note that $L$ itself is the restriction of $\Pi$ to the functions $\mathbf{f}$ satisfying $\Gamma^{\prime} \mathbf{f}=T \Gamma \mathbf{f}, T=\operatorname{diag}(\varepsilon(\boldsymbol{\alpha}))$. This implies

Proposition 2. The spin-orbit Hamiltonian L is self-adjoint.

To carry out the spectral analysis of $L$ it is useful to relate the resolvents of $L$ and $D$ by Krein's resolvent formula [21],

$$
(D-E)^{-1}-(L-E)^{-1}=\gamma(E)[M(E)-T]^{-1} \gamma^{*}(\bar{E})
$$

where $E \notin \operatorname{spec} L \cup \operatorname{spec} D$ and the maps $\gamma(E)$ and $M(E)$ are defined as follows. For a given $E \notin \operatorname{spec} D$ and $\boldsymbol{\xi} \in \ell^{2}\left(\mathscr{V}, \mathbb{C}^{2}\right)$, the function $\gamma(E) \boldsymbol{\xi}=\left(\mathbf{f}_{\boldsymbol{\alpha} \boldsymbol{\beta}}\right)$ is the solution to $(\Pi-E) \mathbf{f}=\mathbf{0}$ satisfying $\Gamma \mathbf{f}=\boldsymbol{\xi}$. The map $M(E): \ell^{2}\left(\mathscr{V}, \mathbb{C}^{2}\right) \rightarrow \ell^{2}\left(\mathscr{V}, \mathbb{C}^{2}\right)$ 
is given by $M(E)=\Gamma^{\prime} \gamma(E)$. A direct consequence of Eq. (11) is the relationship

$$
\operatorname{spec} L \backslash \operatorname{spec} D=\{E \notin \operatorname{spec} D: 0 \in \operatorname{spec}(M(E)-T)\} .
$$

Moreover, $E \notin \operatorname{spec} D$ is an eigenvalue of $L$ iff 0 is an eigenvalue of $M(E)-T$, and $\gamma(E) \operatorname{ker}(M(E)-T)$ is the corresponding eigensubspace [26].

Denote by $\widetilde{D}_{\boldsymbol{\alpha} \boldsymbol{\beta}}$ the self-adjoint operator in $L^{2}\left[0, l_{\boldsymbol{\alpha} \boldsymbol{\beta}}\right]$ acting as $g_{\boldsymbol{\alpha} \boldsymbol{\beta}} \mapsto-g_{\boldsymbol{\alpha} \boldsymbol{\beta}}^{\prime \prime}+$ $\left(U_{\boldsymbol{\alpha} \boldsymbol{\beta}}-k_{R}^{2}\right) g_{\boldsymbol{\alpha} \boldsymbol{\beta}}$ on functions $g_{\boldsymbol{\alpha} \boldsymbol{\beta}} \in H^{2}\left[0, l_{\boldsymbol{\alpha} \boldsymbol{\beta}}\right]$ satisfying the Dirichlet boundary condition, $g_{\alpha \beta}(0)=g_{\alpha \beta}\left(l_{\alpha \beta}\right)$. Note that the operators $\Theta_{\alpha \beta}^{*} D_{\alpha \beta} \Theta_{\alpha \beta}$ are of the form $\widetilde{D}_{\alpha \beta} \oplus \widetilde{D}_{\alpha \beta}$. In particular, the spectra of $D_{\alpha \beta}$ coincide with those of $\widetilde{D}_{\alpha \beta}$ and are discrete sets, and $\operatorname{spec} D=\overline{\bigcup_{\boldsymbol{\alpha} \boldsymbol{\beta} \in \mathscr{E}} \operatorname{spec} \widetilde{D}_{\boldsymbol{\alpha} \boldsymbol{\beta}}}$.

Eq. (12) shows that the spectrum of $L$ outside $\operatorname{spec} D$ is completely described in terms of $M(E)$. The question whether $\operatorname{spec} D$ or some parts of it enter to the spectrum of $L$ must be analyzed individually taking into account the magnetic and spin parameters and the topological properties of the graph.

Therefore, to carry out the spectral analysis for $L$ it is useful to calculate the map $M(E)$. This can be done in terms of special (scalar) solutions to the equation

$$
-y^{\prime \prime}+U_{\boldsymbol{\alpha} \boldsymbol{\beta}} y=z y, \quad z \in \mathbb{C} .
$$

Namely, denote by $s_{\boldsymbol{\alpha} \beta}$ and $c_{\boldsymbol{\alpha} \beta}$ the uniquely determined solutions of (13) satisfying the boundary conditions

$$
s_{\boldsymbol{\alpha} \boldsymbol{\beta}}(0 ; z)=c_{\boldsymbol{\alpha} \boldsymbol{\beta}}^{\prime}(0 ; z)=0, \quad s_{\boldsymbol{\alpha} \boldsymbol{\beta}}^{\prime}(0 ; z)=c_{\boldsymbol{\alpha} \boldsymbol{\beta}}(0 ; z)=1 .
$$

Now let $\boldsymbol{\xi} \in \ell^{2}\left(\mathscr{V}, \mathbb{C}^{2}\right)$. To find $\gamma(E) \boldsymbol{\xi}=:\left(\mathbf{f}_{\boldsymbol{\alpha} \boldsymbol{\beta}}\right)$ we need to solve the boundary value problems

$$
\begin{gathered}
{\left[\left(\mathrm{i} \frac{\mathrm{d}}{\mathrm{d} t}+a_{\boldsymbol{\alpha} \boldsymbol{\beta}}+k_{R} \sigma_{\boldsymbol{\alpha} \boldsymbol{\beta}}\right)^{2}+U_{\boldsymbol{\alpha} \boldsymbol{\beta}}-k_{R}^{2}\right] \mathbf{f}_{\boldsymbol{\alpha} \boldsymbol{\beta}}=E \mathbf{f}_{\boldsymbol{\alpha} \boldsymbol{\beta}},} \\
\mathbf{f}_{\boldsymbol{\alpha} \boldsymbol{\beta}}(0)=\boldsymbol{\xi}(\boldsymbol{\alpha}), \quad \mathbf{f}_{\boldsymbol{\alpha} \boldsymbol{\beta}}\left(l_{\boldsymbol{\alpha} \boldsymbol{\beta}}\right)=\boldsymbol{\xi}(\boldsymbol{\beta}) .
\end{gathered}
$$

Writing $\mathbf{f}_{\alpha \beta}:=\Theta_{\alpha \beta} \mathbf{g}_{\alpha \beta}$, where $\Theta_{\alpha \beta}$ is the unitary transformation from $[8$, we rewrite (14) as a boundary value problem for $\mathbf{g}_{\alpha \boldsymbol{\beta}}$,

$$
\begin{gathered}
-\mathbf{g}_{\boldsymbol{\alpha} \boldsymbol{\beta}}^{\prime \prime}+U_{\boldsymbol{\alpha} \boldsymbol{\beta}} \mathbf{g}_{\boldsymbol{\alpha} \boldsymbol{\beta}}=\left(E+k_{R}^{2}\right) \mathbf{g}_{\boldsymbol{\alpha} \boldsymbol{\beta}}, \\
\mathbf{g}_{\boldsymbol{\alpha} \boldsymbol{\beta}}(0)=\boldsymbol{\xi}(\boldsymbol{\alpha}), \quad \mathbf{g}_{\boldsymbol{\alpha} \boldsymbol{\beta}}\left(l_{\boldsymbol{\alpha} \boldsymbol{\beta}}\right)=\tau_{\boldsymbol{\alpha} \boldsymbol{\beta}}^{*} \boldsymbol{\xi}(\boldsymbol{\beta}) .
\end{gathered}
$$

The solution to (15) takes the form

$$
\begin{aligned}
\mathbf{g}_{\boldsymbol{\alpha} \boldsymbol{\beta}}(t)=\frac{s_{\boldsymbol{\alpha} \boldsymbol{\beta}}\left(t ; E+k_{R}^{2}\right)}{s_{\boldsymbol{\alpha} \boldsymbol{\beta}}\left(l_{\boldsymbol{\alpha} \boldsymbol{\beta}} ; E+k_{R}^{2}\right)} \cdot\left[\tau_{\boldsymbol{\alpha} \boldsymbol{\beta}}^{*} \boldsymbol{\xi}(\boldsymbol{\beta})-c_{\boldsymbol{\alpha} \boldsymbol{\beta}}\left(l_{\boldsymbol{\alpha} \boldsymbol{\beta}} ; E\right.\right. & \left.\left.+k_{R}^{2}\right) \boldsymbol{\xi}(\boldsymbol{\alpha})\right] \\
& +c_{\boldsymbol{\alpha} \boldsymbol{\beta}}\left(t ; E+k_{R}^{2}\right) \boldsymbol{\xi}(\boldsymbol{\alpha}) .
\end{aligned}
$$


Now we have

$$
\begin{aligned}
\mathbf{g}_{\boldsymbol{\alpha} \boldsymbol{\beta}}^{\prime}(0) & =\frac{1}{s_{\boldsymbol{\alpha} \boldsymbol{\beta}}\left(l_{\boldsymbol{\alpha} \boldsymbol{\beta}} ; E+k_{R}^{2}\right)}\left[\tau_{\boldsymbol{\alpha} \boldsymbol{\beta}}^{*} \boldsymbol{\xi}(\boldsymbol{\beta})-c_{\boldsymbol{\alpha} \boldsymbol{\beta}}\left(l_{\boldsymbol{\alpha} \boldsymbol{\beta}} ; E+k_{R}^{2}\right) \boldsymbol{\xi}(\boldsymbol{\alpha})\right], \\
\mathbf{g}_{\boldsymbol{\alpha} \boldsymbol{\beta}}^{\prime}\left(l_{\boldsymbol{\alpha} \boldsymbol{\beta}}\right) & =\frac{1}{s_{\boldsymbol{\alpha} \boldsymbol{\beta}}\left(l_{\boldsymbol{\alpha} \boldsymbol{\beta}} ; E+k_{R}^{2}\right)}\left[s_{\boldsymbol{\alpha} \boldsymbol{\beta}}^{\prime}\left(l_{\boldsymbol{\alpha} \boldsymbol{\beta}} ; E+k_{R}^{2}\right) \tau_{\boldsymbol{\alpha} \boldsymbol{\beta}}^{*} \boldsymbol{\xi}(\boldsymbol{\beta})-\boldsymbol{\xi}(\boldsymbol{\alpha})\right] .
\end{aligned}
$$

Noting that

$$
\mathbf{f}^{\prime}(\boldsymbol{\alpha})=\sum_{\boldsymbol{\alpha} \boldsymbol{\beta} \in \mathscr{E}} \mathbf{g}_{\boldsymbol{\alpha} \boldsymbol{\beta}}^{\prime}-\sum_{\boldsymbol{\beta} \boldsymbol{\alpha} \in \mathscr{E}} \tau_{\beta \alpha} \mathbf{g}_{\boldsymbol{\alpha} \boldsymbol{\beta}}^{\prime}\left(l_{\boldsymbol{\alpha} \boldsymbol{\beta}}\right)
$$

we arrive at

$$
\begin{aligned}
M(E) \boldsymbol{\xi}(\boldsymbol{\alpha})= & \sum_{\boldsymbol{\alpha} \boldsymbol{\beta} \in \mathscr{E}} \frac{1}{s_{\boldsymbol{\alpha} \boldsymbol{\beta}}\left(l_{\boldsymbol{\alpha} \boldsymbol{\beta}} ; E+k_{R}^{2}\right)} \tau_{\boldsymbol{\alpha} \boldsymbol{\beta}}^{*} \boldsymbol{\xi}(\boldsymbol{\beta}) \\
& +\sum_{\boldsymbol{\beta} \boldsymbol{\alpha} \in \mathscr{E}} \frac{1}{s_{\boldsymbol{\beta} \boldsymbol{\alpha}}\left(l_{\boldsymbol{\beta} \boldsymbol{\alpha}} ; E+k_{R}^{2}\right)} \tau_{\boldsymbol{\beta} \boldsymbol{\alpha}} \boldsymbol{\xi}(\boldsymbol{\beta}) \\
& -\left[\sum_{\boldsymbol{\alpha} \boldsymbol{\beta} \in \mathscr{E}} \frac{c_{\boldsymbol{\alpha} \boldsymbol{\beta}}\left(l_{\boldsymbol{\alpha} \boldsymbol{\beta}} ; E+k_{R}^{2}\right)}{s_{\boldsymbol{\alpha} \boldsymbol{\beta}}\left(l_{\boldsymbol{\alpha} \boldsymbol{\beta}} ; E+k_{R}^{2}\right)}+\sum_{\boldsymbol{\beta} \boldsymbol{\alpha} \in \mathscr{E}} \frac{s_{\boldsymbol{\beta} \boldsymbol{\alpha}}^{\prime}\left(l_{\boldsymbol{\beta} \boldsymbol{\alpha}} ; E+k_{R}^{2}\right)}{s_{\boldsymbol{\beta} \boldsymbol{\alpha}}\left(l_{\boldsymbol{\beta} \boldsymbol{\alpha}} ; E+k_{R}^{2}\right)}\right] \boldsymbol{\xi}(\boldsymbol{\alpha}) .
\end{aligned}
$$

Using Krein's resolvent formula (11) we come to

Theorem 3. The set $\operatorname{spec} L \backslash \operatorname{spec} D$ consists exactly of the real numbers $E$ such that $0 \in \operatorname{spec}[M(E)-T]$, where $M(E)$ and $T$ are operators in $\ell^{2}\left(\mathscr{V}, \mathbb{C}^{2}\right), M(E)$ is given by (18) and $T=\operatorname{diag}(\varepsilon(\boldsymbol{\alpha}))$. Moreover, such $E$ is an eigenvalue of $L$ iff 0 is an eigenvalue of $M(E)-T$, and $\gamma(E) \operatorname{ker}(M(E)-T)$ is the corresponding eigenspace.

We remark that in the above calculations it does not matter whether $\boldsymbol{\xi}$ is in $\ell^{2}$ or not. Actually, all the construction hold for any set of vectors $\boldsymbol{\xi}(\boldsymbol{\alpha}) \in \mathbb{C}^{2}$, $\boldsymbol{\alpha} \in \mathscr{V}$. This observation can be formulated as follows:

Theorem 4. For $E \notin \operatorname{spec} D$, any continuous solution $\mathbf{f}$ to $(L-E) \mathbf{f}=0$ has the form

$$
\begin{aligned}
\mathbf{f}_{\boldsymbol{\alpha} \boldsymbol{\beta}}(t)=\frac{s_{\boldsymbol{\alpha} \boldsymbol{\beta}}\left(t ; E+k_{R}^{2}\right)}{s_{\boldsymbol{\alpha} \boldsymbol{\beta}}\left(l_{\boldsymbol{\alpha} \boldsymbol{\beta}} ; E+k_{R}^{2}\right)} \cdot \Theta_{\boldsymbol{\alpha} \boldsymbol{\beta}}\left[\tau_{\boldsymbol{\alpha} \boldsymbol{\beta}}^{*} \mathbf{f}(\boldsymbol{\beta})-c_{\boldsymbol{\alpha} \boldsymbol{\beta}}\left(l_{\boldsymbol{\alpha} \boldsymbol{\beta}} ; E+k_{R}^{2}\right) \mathbf{f}(\boldsymbol{\alpha})\right] \\
+\Theta_{\boldsymbol{\alpha} \boldsymbol{\beta}} c_{\boldsymbol{\alpha} \boldsymbol{\beta}}\left(t ; E+k_{R}^{2}\right) \mathbf{f}(\boldsymbol{\alpha}) .
\end{aligned}
$$

Such a function satisfies the boundary conditions (6b) iff

$$
\begin{aligned}
\sum_{\boldsymbol{\alpha} \boldsymbol{\beta} \in \mathscr{E}} \frac{1}{s_{\boldsymbol{\alpha} \boldsymbol{\beta}}\left(l_{\boldsymbol{\alpha} \boldsymbol{\beta}} ; E+k_{R}^{2}\right)} \tau_{\boldsymbol{\alpha} \boldsymbol{\beta}}^{*} \mathbf{f}(\boldsymbol{\beta})+\sum_{\boldsymbol{\beta} \boldsymbol{\alpha} \in \mathscr{E}} \frac{1}{s_{\boldsymbol{\beta} \boldsymbol{\alpha}}\left(l_{\boldsymbol{\beta} \boldsymbol{\alpha}} ; E+k_{R}^{2}\right)} \tau_{\boldsymbol{\beta} \boldsymbol{\alpha}} \mathbf{f}(\boldsymbol{\beta}) \\
=\left[\sum_{\boldsymbol{\alpha} \boldsymbol{\beta} \in \mathscr{E}} \frac{c_{\boldsymbol{\alpha} \boldsymbol{\beta}}\left(l_{\boldsymbol{\alpha} \boldsymbol{\beta}} ; E+k_{R}^{2}\right)}{s_{\boldsymbol{\alpha} \boldsymbol{\beta}}\left(l_{\boldsymbol{\alpha} \boldsymbol{\beta}} ; E+k_{R}^{2}\right)}+\sum_{\boldsymbol{\beta} \boldsymbol{\alpha} \in \mathscr{E}} \frac{s_{\boldsymbol{\beta} \boldsymbol{\alpha}}^{\prime}\left(l_{\boldsymbol{\beta} \boldsymbol{\alpha}} ; E+k_{R}^{2}\right)}{s_{\boldsymbol{\beta} \boldsymbol{\alpha}}\left(l_{\boldsymbol{\beta} \boldsymbol{\alpha}} ; E+k_{R}^{2}\right)}+\varepsilon(\boldsymbol{\alpha})\right] \mathbf{f}(\boldsymbol{\alpha}) .
\end{aligned}
$$


Such an $\mathbf{f}$ is an eigenfunction of $L$ (i.e. belongs to $\left.L^{2}\right)$ iff $(\mathbf{f}(\boldsymbol{\alpha}))_{\boldsymbol{\alpha} \in \mathscr{V}} \in \ell^{2}\left(\mathscr{V}, \mathbb{C}^{2}\right)$.

Note that similar formulas for more simple situations were obtained earlier e.g.in $[3,5,8,22,23,32]$.

The expression (18) can be simplified significantly if all the edges are the same, i.e. if $l_{\boldsymbol{\alpha} \beta} \equiv l$ and $U_{\boldsymbol{\alpha} \boldsymbol{\beta}} \equiv U, s_{\boldsymbol{\alpha} \beta}=s, c_{\boldsymbol{\alpha} \beta}=c$ for all $\boldsymbol{\alpha} \boldsymbol{\beta} \in \mathscr{E}$. Note that in this case the spectrum of $D$ coincides with the Dirichlet spectrum of the operator $\widetilde{D}:=-\mathrm{d}^{2} / \mathrm{d} t^{2}+U-k_{R}^{2}$ on the segment $[0, l]$ and hence is a discrete set. We have

$$
\begin{aligned}
M(E) \boldsymbol{\xi}(\boldsymbol{\alpha})= & \frac{1}{s\left(l ; E+k_{R}^{2}\right)}\left\{\left[\sum_{\boldsymbol{\alpha} \boldsymbol{\beta} \in \mathscr{E}} \tau_{\boldsymbol{\alpha} \boldsymbol{\beta}}^{*} \boldsymbol{\xi}(\boldsymbol{\beta})+\sum_{\boldsymbol{\beta} \boldsymbol{\alpha} \in \mathscr{E}} \tau_{\boldsymbol{\beta} \boldsymbol{\alpha}} \boldsymbol{\xi}(\boldsymbol{\beta})\right]\right. \\
& \left.-\left[\operatorname{outdeg} \boldsymbol{\alpha} c\left(l ; E+k_{R}^{2}\right)+\operatorname{indeg} \boldsymbol{\alpha} s^{\prime}\left(l ; E+k_{R}^{2}\right)\right] \boldsymbol{\xi}(\boldsymbol{\alpha})\right\} .
\end{aligned}
$$

Even this expression admits further simplifications.

Proposition 5. Assume that all edges are identical, $l_{\alpha \beta} \equiv l, U_{\alpha \beta} \equiv U, U$ is even, $U(t) \equiv U(l-x)$, and the coupling constants $\varepsilon(\boldsymbol{\alpha})$ are of the form $\varepsilon(\boldsymbol{\alpha})=\operatorname{deg} \boldsymbol{\alpha} \varepsilon$, then $\operatorname{spec} L \backslash \operatorname{spec} \widetilde{D}=t_{\varepsilon}^{-1}(\operatorname{spec} \Delta)$, where $t_{\varepsilon}(E)=c\left(l ; E+k_{R}^{2}\right)+\varepsilon s\left(l ; E+k_{R}^{2}\right)$ and $\Delta$ is the discrete Hamiltonian,

$$
\Delta \boldsymbol{\xi}(\boldsymbol{\alpha})=\frac{1}{\operatorname{deg} \boldsymbol{\alpha}}\left(\sum_{\boldsymbol{\alpha} \in \mathscr{E}} \tau_{\boldsymbol{\alpha} \beta}^{*} \boldsymbol{\xi}(\boldsymbol{\beta})+\sum_{\boldsymbol{\beta} \boldsymbol{\alpha} \in \mathscr{E}} \tau_{\beta \alpha} \boldsymbol{\xi}(\boldsymbol{\beta})\right),
$$

acting on the space $\ell^{2}\left(\mathscr{V}, \mathbb{C}^{2} ; \mathrm{deg}\right)$ with the scalar product

$$
\langle\boldsymbol{\xi}, \boldsymbol{\eta}\rangle_{\operatorname{deg}}=\sum_{\boldsymbol{\alpha} \in \mathscr{V}} \operatorname{deg} \boldsymbol{\alpha} \cdot \overline{\boldsymbol{\xi}(\boldsymbol{\alpha})} \boldsymbol{\eta}(\boldsymbol{\alpha}) .
$$

Proof. If the potential $U$ is even, one has $c\left(l ; E+k_{R}^{2}\right) \equiv s^{\prime}\left(l ; E+k_{R}^{2}\right):=t(E)$, see e.g. [44], hence

$$
M(E)-T=\frac{1}{s\left(l ; E+k_{R}^{2}\right)}\left[\widetilde{\Delta}-t_{\mathcal{E}}(E) \operatorname{deg}\right], \quad \operatorname{deg}=\operatorname{diag}(\operatorname{deg} \boldsymbol{\alpha}),
$$

where $\widetilde{\Delta}$ is the discrete Hamiltonian in $\ell^{2}\left(\mathscr{V}, \mathbb{C}^{2}\right)$,

$$
\widetilde{\Delta} \boldsymbol{\xi}(\boldsymbol{\alpha})=\sum_{\boldsymbol{\alpha} \boldsymbol{\beta} \in \mathscr{E}} \tau_{\alpha \beta}^{*} \boldsymbol{\xi}(\boldsymbol{\beta})+\sum_{\boldsymbol{\beta} \boldsymbol{\alpha} \in \mathscr{E}} \tau_{\beta \alpha} \boldsymbol{\xi}(\boldsymbol{\beta}) .
$$

The condition $0 \in \operatorname{spec}[M(E)-T]$ takes the form $0 \in \operatorname{spec}\left[\widetilde{\Delta}-t_{\varepsilon}(E) \operatorname{deg}\right]$ in $\ell^{2}\left(\mathscr{V}, \mathbb{C}^{2}\right)$, which is equivalent to $0 \in \operatorname{spec}\left[\Delta-t_{\mathcal{\varepsilon}}(E)\right]$ in $\ell^{2}\left(\mathscr{V}, \mathbb{C}^{2} ; \operatorname{deg}\right)$. 


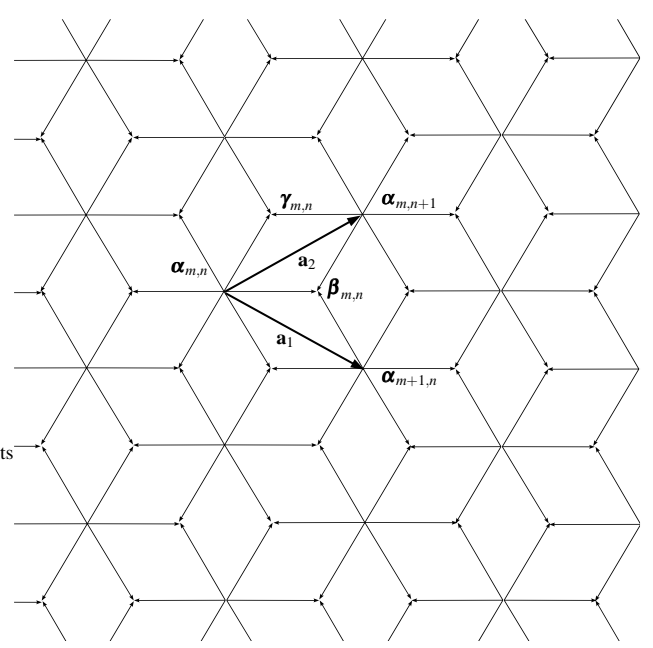

Figure 1: A finite piece of $T_{3}$-lattice

Proposition 5 shows that the spectral problem for a class of quantum graphs reduces to the study of the tight-binding Hamiltonian $\Delta$. In the case $U \equiv$ 0 and $\varepsilon(\boldsymbol{\alpha}) \equiv 0$ one has $t_{\varepsilon}(E)=\cos \sqrt{E+k_{R}^{2}}$, and we arrive at $\operatorname{spec} L=$ $\operatorname{Arccos}^{2} \operatorname{spec} \Delta-k_{R}^{2}$ (up to the discrete set $\operatorname{spec} \widetilde{D}$ ), which is exactly the formula connecting the network and the tight-binding spectra in the de Gennes-Alexander model of superconductivity [4]. For scalar situation, an analogue of this correspondence was given e.g. in [5] for the Laplacian on compact graphs, in [16] for the Laplacian on non-compact graphs, and in [44] for more general Schrödinger operators. At the same time, proposition 5] does not exhaust all possibilities of such a reduction, i.e. the reduction to a discrete Hamiltonian is possible also for some non-even $U$. (Such questions will be discussed in greater detail in [45].) One of such situations will be discussed in the next section.

\section{Spectrum of $T_{3}$-lattice}

\subsection{Description of the lattice}

In this section, we consider the spectral problem for a quantum graph whose underlying structure is the so-called $T_{3}$-lattice (see figure1). The nodes are the points $\boldsymbol{\alpha}_{m, n}, \boldsymbol{\beta}_{m, n}, \gamma_{m, n}$ with $\boldsymbol{\alpha}_{m, n}=m \mathbf{a}_{1}+n \mathbf{a}_{2}, \mathbf{a}_{1}=\left(\frac{3}{2},-\frac{\sqrt{3}}{2}, 0\right), \mathbf{a}_{2}=\left(\frac{3}{2}, \frac{\sqrt{3}}{2}, 0\right)$, 


$$
\begin{gathered}
\boldsymbol{\beta}_{m, n}=\boldsymbol{\alpha}_{m, n}+(1,0,0), \gamma_{m, n}=\boldsymbol{\alpha}_{m, n}+\left(\frac{1}{2}, \frac{\sqrt{3}}{2}, 0\right), m, n \in \mathbb{Z}, \text { i.e. } \\
\boldsymbol{\alpha}_{m, n}=\left(\frac{3(m+n)}{2}, \frac{\sqrt{3}(n-m)}{2}, 0\right), \quad \boldsymbol{\beta}_{m, n}=\left(\frac{3(m+n)+2}{2}, \frac{\sqrt{3}(n-m)}{2}, 0\right), \\
\gamma_{m, n}=\left(\frac{3(m+n)+1}{2}, \frac{\sqrt{3}(n-m+1)}{2}, 0\right) .
\end{gathered}
$$

The edges are

$$
\begin{array}{lll}
e_{m, n, 1}=\boldsymbol{\alpha}_{m, n} \boldsymbol{\gamma}_{m, n}, & e_{m, n, 2}=\boldsymbol{\alpha}_{m, n} \boldsymbol{\beta}_{m-1, n}, & e_{m, n, 3}=\boldsymbol{\alpha}_{m, n} \boldsymbol{\gamma}_{m, n-1}, \\
e_{m, n, 4}=\boldsymbol{\alpha}_{m, n} \boldsymbol{\beta}_{m, n-1}, & e_{m, n, 5}=\boldsymbol{\alpha}_{m, n} \boldsymbol{\gamma}_{m+1, n-1}, & e_{m, n, 6}=\boldsymbol{\alpha}_{m, n} \boldsymbol{\beta}_{m, n}
\end{array}
$$

All the edges have the length 1. The direction vectors of $e_{m, n, j}$ are $\mathbf{e}_{j}=$ $\left(\cos \frac{\pi j}{3}, \sin \frac{\pi j}{3}, 0\right), j=1, \ldots, 6$.

\subsection{Reduction to tight-binding Hamiltonian}

We will assume that the system is subjected to the following external interactions. On each edge there is the same potential $U \in L^{2}[0,1]$. The lattice is subjected to the uniform magnetic field $\mathbf{B}=(0,0,2 \pi \xi)$ orthogonal to the plane, and the magnetic vector potential in the symmetric gauge is $\mathbf{A}(\mathbf{x})=\left(-\pi \xi_{x_{2}}, \pi \xi_{x_{1}, 0}\right)$. In what follows we use the magnetic parameter $\omega=\frac{\pi \xi \sqrt{3}}{2}$ expressing the magnetic flux through the elementary rhombus (for example, $\boldsymbol{\alpha}_{m, n} \boldsymbol{\beta}_{m, n} \boldsymbol{\alpha}_{m, n+1} \boldsymbol{\gamma}_{m n}$ ).

The external magnetic field implies non-trivial magnetic potentials on $e_{m, n, j}$, $a_{m, n, j}=\frac{1}{2}\left\langle\mathbf{B} \times \boldsymbol{\alpha}_{m, n}, \mathbf{e}_{j}\right\rangle$,

$$
\begin{aligned}
& a_{m, n, 1}=\omega(2 m+n), \quad a_{m, n, 2}=\omega(m+2 n), \quad a_{m, n, 3}=\omega(n-m), \\
& a_{m, n, 4}=-\omega(2 m+n), \quad a_{m, n, 5}=-\omega(m+2 n), \quad a_{m, n, 3}=\omega(m-n) .
\end{aligned}
$$

The dynamics along $e_{m, n, j}$ is described by the differential expression

$$
\begin{gathered}
L_{m, n, j}=\left(\mathrm{i} \frac{\mathrm{d}}{\mathrm{d} t}+a_{m, n, j}+k_{R} \sigma_{m, n, j}\right)^{2}+U-k_{R}^{2} \\
\sigma_{m, n, j}=\left(\begin{array}{cc}
0 & e_{j 2}+i e_{j 1} \\
e_{j 2}-i e_{j 1} & 0
\end{array}\right) \equiv\left(\begin{array}{cc}
0 & \exp \left[\mathrm{i}\left(\frac{\pi}{2}-\frac{\pi j}{3}\right)\right] \\
\exp \left[\mathrm{i}\left(\frac{\pi j}{3}-\frac{\pi}{2}\right)\right] & 0
\end{array}\right), \\
m, n \in \mathbb{Z}, \quad j \in\{1, \ldots, 6\} .
\end{gathered}
$$


Here $e_{j k}$ are the components of the vectors $\mathbf{e}_{j}$ and $k_{R}$ is the Rashba constant. We consider boundary conditions of the form (6) at all the nodes assuming that there are only two types of coupling constants: $\lambda:=\varepsilon\left(\boldsymbol{\alpha}_{m, n}\right)$ and $\mu:=\varepsilon\left(\boldsymbol{\beta}_{m, n}\right)=$ $\varepsilon\left(\gamma_{m, n}\right)$.

The corresponding matrices $\tau_{m, n, j}$ from Eq. (10), $\tau_{m, n, j}=\exp \left[\mathrm{i}\left(a_{m, n, j}+\right.\right.$ $\left.\left.k_{R} \sigma_{m, n, j}\right)\right]$, are as follows:

$$
\begin{aligned}
& \tau_{m, n, 1}=\tau_{m, n, 4}^{*}=\mathrm{e}^{\mathrm{i} \omega(2 m+n)}\left\{\cos k_{R}+\mathrm{i} \sin k_{R}\left(\begin{array}{cc}
0 & \mathrm{e}^{\mathrm{i} \pi / 6} \\
\mathrm{e}^{-\mathrm{i} \pi / 6} & 0
\end{array}\right)\right\}, \\
& \tau_{m, n, 2}=\tau_{m, n, 5}^{*}=\mathrm{e}^{\mathrm{i} \omega(m+2 n)}\left\{\cos k_{R}+\mathrm{i} \sin k_{R}\left(\begin{array}{cc}
0 & \mathrm{e}^{-\mathrm{i} \pi / 6} \\
\mathrm{e}^{\mathrm{i} \pi / 6} & 0
\end{array}\right)\right\}, \\
& \tau_{m, n, 3}=\tau_{m, n, 6}^{*}=\mathrm{e}^{\mathrm{i} \omega(n-m)}\left\{\cos k_{R}+\mathrm{i} \sin k_{R}\left(\begin{array}{cc}
0 & \mathrm{e}^{-\mathrm{i} \pi / 2} \\
\mathrm{e}^{\mathrm{i} \pi / 2} & 0
\end{array}\right)\right\} .
\end{aligned}
$$

Clearly, for any $m, n \in \mathbb{Z}$ one has

$$
\begin{gathered}
\text { outdeg } \boldsymbol{\alpha}_{m, n}=6, \quad \operatorname{indeg} \boldsymbol{\beta}_{m, n}=\operatorname{indeg} \gamma_{m, n}=3, \\
\text { indeg } \boldsymbol{\alpha}_{m, n}=\operatorname{outdeg} \boldsymbol{\beta}_{m, n}=\operatorname{outdeg} \gamma_{m, n}=0 .
\end{gathered}
$$

For the subsequent analysis we use the fact that the lattice is bipartite. Represent the set of nodes as the disjoint union $\mathscr{V}=\mathscr{V}_{0} \cup \mathscr{V}_{1}, \mathscr{V}_{0}=\left\{\boldsymbol{\alpha}_{m, n}\right\}, \mathscr{V}_{1}=$ $\left\{\boldsymbol{\beta}_{m, n}\right\} \cup\left\{\gamma_{m, n}\right\}$. Clearly, for the set of edges one has $\mathscr{E} \subset \mathscr{V}_{0} \times \mathscr{V}_{1}$. With respect the the decomposition $\ell^{2}\left(\mathscr{V}, \mathbb{C}^{2}\right)=\ell^{2}\left(\mathscr{V}_{0}, \mathbb{C}^{2}\right) \oplus \ell^{2}\left(\mathscr{V}_{1}, \mathbb{C}^{2}\right)$ the operator $T$ in theorem 3 takes the block-diagonal form,

$$
T=\left(\begin{array}{ll}
\lambda & 0 \\
0 & \mu
\end{array}\right) .
$$

Using the above decomposition and Eq. (19), we rewrite $M(E)-T$ as

$$
\begin{gathered}
M(E)-T=\frac{1}{s\left(1 ; E+k_{R}^{2}\right)}\left(\begin{array}{cc}
-a(E) & A^{*} \\
A & -b(E)
\end{array}\right), \\
a(E)=6 c\left(1 ; E+k_{R}^{2}\right)+\lambda s\left(1 ; E+k_{R}^{2}\right), \\
b(E)=3 s^{\prime}\left(1 ; E+k_{R}^{2}\right)+\mu s\left(1 ; E+k_{R}^{2}\right) .
\end{gathered}
$$

where

$$
\begin{aligned}
A \mathbf{f}\left(\boldsymbol{\beta}_{m, n}\right)= & \tau_{m+1, n, 2} \mathbf{f}\left(\boldsymbol{\alpha}_{m+1, n}\right)+\tau_{m, n+1,4} \mathbf{f}\left(\boldsymbol{\alpha}_{m, n+1}\right)+\tau_{m, n, 6} \mathbf{f}\left(\boldsymbol{\alpha}_{m, n}\right), \\
A \mathbf{f}\left(\boldsymbol{\gamma}_{m, n}\right)= & \tau_{m, n, 1} \mathbf{f}\left(\boldsymbol{\alpha}_{m, n}\right)+\tau_{m, n+1,3} \mathbf{f}\left(\boldsymbol{\alpha}_{m, n+1}\right)+\tau_{m-1, n+1,5} \mathbf{f}\left(\boldsymbol{\alpha}_{m-1, n+1}\right), \\
A^{*} \mathbf{f}\left(\boldsymbol{\alpha}_{m, n}\right)= & \tau_{m, n, 1}^{*} \mathbf{f}\left(\boldsymbol{\gamma}_{m, n}\right)+\tau_{m, n, 2}^{*} \mathbf{f}\left(\boldsymbol{\beta}_{m-1, n}\right)+\tau_{m, n, 3}^{*} \mathbf{f}\left(\boldsymbol{\gamma}_{m, n-1}\right) \\
& +\tau_{m, n, 4}^{*} \mathbf{f}\left(\boldsymbol{\beta}_{m, n-1}\right)+\tau_{m, n, 5}^{*} \mathbf{f}\left(\boldsymbol{\gamma}_{m+1, n-1}\right)+\tau_{m, n, 6}^{*} \mathbf{f}\left(\boldsymbol{\beta}_{m, n}\right) .
\end{aligned}
$$


The operator $A^{*}, A^{*}: \ell^{2}\left(\mathscr{V}_{1}, \mathbb{C}^{2}\right) \rightarrow \ell^{2}\left(\mathscr{V}_{0}, \mathbb{C}^{2}\right)$ is adjoint to $A, A: \ell^{2}\left(\mathscr{V}_{0}, \mathbb{C}^{2}\right) \rightarrow$ $\ell^{2}\left(\mathscr{V}_{1}, \mathbb{C}^{2}\right)$. Using theorem 3 we write the condition $E \in \operatorname{spec} L$ or, equivalently, $0 \in \operatorname{spec}(M(E)-T)$, as

$$
\frac{a(E)+b(E)}{2} \in \operatorname{spec}\left[\frac{b(E)-a(E)}{2}\left(\begin{array}{cc}
1 & 0 \\
0 & -1
\end{array}\right)+\left(\begin{array}{cc}
0 & A^{*} \\
A & 0
\end{array}\right)\right] .
$$

Note that $E \notin \operatorname{spec} \widetilde{D}$ in all the above constructions, where $\widetilde{D}$ is the Dirichlet realization of $-\mathrm{d}^{2} / \mathrm{d} t^{2}+U-k_{R}^{2}$ on $[0,1]$. The question whether $\operatorname{spec} \widetilde{D}$ is a part of $\operatorname{spec} L$ or not admits a simple answer in our case.

Lemma 6. For all $\omega$ and $k_{R}$ one has $\operatorname{spec} \widetilde{D} \subset \operatorname{spec} L$.

Proof. Using the Schnol-type arguments, cf. [12,33], it is sufficient to show that for each $E \in \operatorname{spec} \widetilde{D}$ the equation $L \mathbf{f}=E \mathbf{f}$ has a bounded solution $\mathbf{f}$ satisfying the boundary conditions (6). Let $E \in \widetilde{D}$ and $g$ be the corresponding eigenfunction of $\widetilde{D}$. Choose any infinite path $\mathscr{P}$ without intersection on the graph and any non-zero vector $\mathbf{z} \in \mathbb{C}^{2}$. For a fixed $e \in \mathscr{P}$ set $\mathbf{f}_{e}:=\Theta_{e} g \mathbf{z}$, where $\Theta_{e}$ is given by (8). Now extend $\mathbf{f}$ to the whole graph in such a way that (a) $\mathbf{f}_{e}=\mathbf{0}$ for $e \notin \mathscr{P}$ and (b) on each $b \in \mathscr{P}$ one has $\mathbf{f}_{b}=\Theta_{b} g \mathbf{z}_{b}$, where the vectors $\mathbf{z}_{b}$ are chosen in such a way that the boundary conditions (6) are satisfied. By construction, there holds $L \mathbf{f}=E \mathbf{f}$. At the same time, due to the unitarity of the matrices $\tau_{m, n, j}$ the obtained function $\mathbf{f}$ is bounded. This finishes the proof.

\subsection{Supersymmetric analysis}

Eq. (22) is a typical supersymmetric spectral problem. Using proposition 11 and corollary 12 in Appendix one easily sees that the set $\Sigma$ of $E$ for which the condition (22) is satisfied is the union $\Sigma=\Sigma_{1} \cup \Sigma_{2} \cup \Sigma_{3}$,

$$
\begin{aligned}
\Sigma_{1}=\left\{E \notin \operatorname{spec} \widetilde{D}: a(E) b(E) \neq 0 \text { and } a(E) b(E) \in \operatorname{spec} A^{*} A,\right\} \\
\Sigma_{2}= \begin{cases}\{E \notin \operatorname{spec} \widetilde{D}: a(E)=0\}, & \text { if } 0 \in \operatorname{spec} A^{*} A, \\
\varnothing, & \text { otherwise, }\end{cases} \\
\Sigma_{3}= \begin{cases}\{E \notin \operatorname{spec} \widetilde{D}: b(E)=0\}, & \text { if } 0 \in \operatorname{spec} A A^{*}, \\
\varnothing, & \text { otherwise. }\end{cases}
\end{aligned}
$$

To summarize,

Proposition 7. $\operatorname{spec} L=\Sigma_{1} \cup \Sigma_{2} \cup \Sigma_{3} \cup \operatorname{spec} \widetilde{D}$. 
Note that the sets $\Sigma_{2}, \Sigma_{3}$, and spec $\widetilde{D}$ are discrete. Therefore, only the set $\Sigma_{1}$ is responsible for the continuous spectrum. Writing $\mathbf{f}\left(\boldsymbol{\alpha}_{m, n}\right)=: \mathbf{f}(m, n)$, we note that $A^{*} A$ is an operator on $\ell^{2}\left(\mathbb{Z}^{2}, \mathbb{C}^{2}\right)$ of the form

$$
\begin{aligned}
A^{*} A \mathbf{f}(m, n)= & 6 \mathbf{f}(m, n) \\
& +\left(\tau_{m, n, 1}^{*} \tau_{m, n+1,3}+\tau_{m, n, 6}^{*} \tau_{m, n+1,4}\right) \mathbf{f}(m, n+1) \\
& +\left(\tau_{m, n, 3}^{*} \tau_{m, n-1,1}+\tau_{m, n, 4}^{*} \tau_{m, n-1,6}\right) \mathbf{f}(m, n-1) \\
& +\left(\tau_{m, n, 5}^{*} \tau_{m+1, n, 3}+\tau_{m, n, 6}^{*} \tau_{m+1, n, 2}\right) \mathbf{f}(m+1, n) \\
& +\left(\tau_{m, n, 2}^{*} \tau_{m-1, n, 6}+\tau_{m, n, 3}^{*} \tau_{m-1, n, 5}\right) \mathbf{f}(m-1, n) \\
& +\left(\tau_{m, n, 1}^{*} \tau_{m-1, n+1,5}+\tau_{m, n, 2}^{*} \tau_{m-1, n+1,4}\right) \mathbf{f}(m-1, n+1) \\
& +\left(\tau_{m, n, 4}^{*} \tau_{m+1, n-1,2}+\tau_{m, n, 5}^{*} \tau_{m+1, n-1,1}\right) \mathbf{f}(m+1, n-1)
\end{aligned}
$$

i.e.

$$
\begin{aligned}
& A^{*} A=6+\cos \omega \cdot \sin 2 k_{R} \cdot \widetilde{\Delta} \\
& \quad+2\left[\cos \omega \cdot \cos ^{2} k_{R}-\sin ^{2} k_{R}\left(\begin{array}{cc}
\cos \left(\omega-\frac{\pi}{3}\right) & 0 \\
0 & \cos \left(\omega+\frac{\pi}{3}\right)
\end{array}\right)\right]\left(\begin{array}{cc}
\Delta & 0 \\
0 & \Delta
\end{array}\right)
\end{aligned}
$$

where $\Delta$ is a spinless operator in $\ell^{2}\left(\mathbb{Z}^{2}\right)$,

$$
\begin{aligned}
\Delta f(m, n) & =\mathrm{e}^{-3 \mathrm{i} \omega m} f(m, n+1)+\mathrm{e}^{3 \mathrm{i} \omega m} f(m, n-1) \\
& +\mathrm{e}^{3 \mathrm{i} \omega n} f(m+1, n)+\mathrm{e}^{-3 \mathrm{i} \omega n} f(m-1, n) \\
& +\mathrm{e}^{-3 \mathrm{i} \omega(m+n)} f(m-1, n+1)+\mathrm{e}^{3 \mathrm{i} \omega(m+n)} f(m+1, n-1),
\end{aligned}
$$

and

$$
\begin{aligned}
\widetilde{\Delta} \mathbf{f}(m, n) & =R_{1} \mathrm{e}^{-3 \mathrm{i} \omega m} \mathbf{f}(m, n+1)+R_{1}^{*} \mathrm{e}^{3 \mathrm{i} \omega m} \mathbf{f}(m, n-1) \\
& +R_{2} \mathrm{e}^{3 \mathrm{i} \omega n} \mathbf{f}(m+1, n)+R_{2}^{*} \mathrm{e}^{-3 \mathrm{i} \omega n} \mathbf{f}(m-1, n) \\
& +R_{3} \mathrm{e}^{-3 \mathrm{i} \omega(m+n)} \mathbf{f}(m-1, n+1)+R_{3}^{*} \mathrm{e}^{3 \mathrm{i} \omega(m+n)} \mathbf{f}(m+1, n-1)
\end{aligned}
$$

with

$$
\begin{gathered}
R_{1}=\left(\begin{array}{cc}
0 & \frac{3}{2}-\frac{\sqrt{3}}{2} \mathrm{i} \\
-\frac{3}{2}-\frac{\sqrt{3}}{2} \mathrm{i} & 0
\end{array}\right), \quad R_{2}=\left(\begin{array}{cc}
0 & \frac{3}{2}+\frac{\sqrt{3}}{2} \mathrm{i} \\
-\frac{3}{2}+\frac{\sqrt{3}}{2} \mathrm{i} & 0
\end{array}\right) \\
R_{3}=\left(\begin{array}{cc}
0 & -\sqrt{3} \mathrm{i} \\
-\sqrt{3} \mathrm{i} & 0
\end{array}\right) .
\end{gathered}
$$

The expression for $A^{*} A$ shows explicitly the contribution of the magnetic and spinorbit parameters to the spectrum. Let us discuss the situations where the spectrum shows certain localization phenomena. 


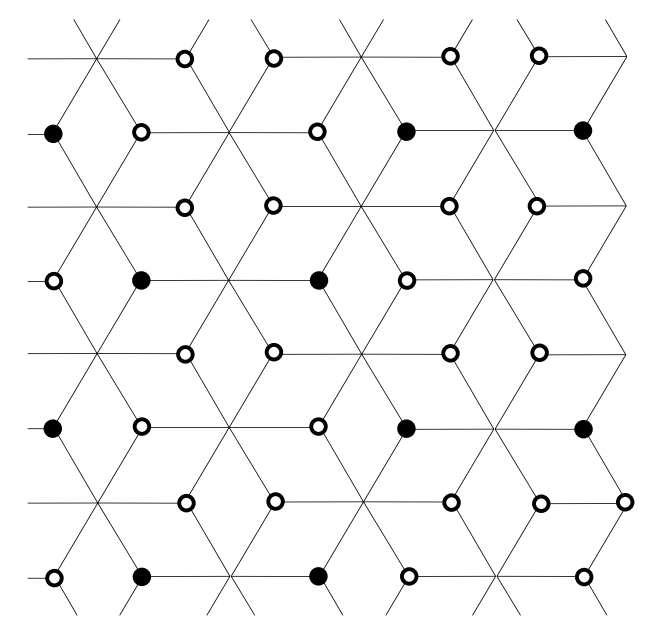

Figure 2: Classification of the nodes $\boldsymbol{\beta}_{m, n}$ and $\boldsymbol{\gamma}_{m, n}$.

\subsection{Magnetic field induced extreme localization}

If the spin-orbit interaction is not taken into account, $k_{R}=0$, then one has $A^{*} A=6+2 \cos \omega(\Delta \oplus \Delta)$. In particular, at $\omega-\frac{\pi}{2} \in \pi \mathbb{Z}$ one has $A^{*} A=6$, i.e. the spectrum of $A^{*} A$ degenerates to a point. If $\omega-\frac{\pi}{2} \in \pi \mathbb{Z}$ but the spin-orbit interaction is non-trivial, similar phenomena occur only at certain values of the Rashba constant, i.e. $k_{R} \in \pi \mathbb{Z}$. For generic values of $k_{R}$, obviously, there are some bands of continuous spectrum.

Let us analyze the sets $\Sigma_{2}$ and $\Sigma_{3}$ for this case, i.e. for $\omega-\frac{\pi}{2} \in \pi \mathbb{Z}$ and $k_{R} \in \pi \mathbb{Z}$. Clearly, the set $\Sigma_{2}$ is empty, as $0 \notin \operatorname{spec} A^{*} A$. Let us look at the operator $A A^{*}$.

Lemma 8. For $\omega-\frac{\pi}{2} \in \pi \mathbb{Z}$ and $k_{R} \in \pi \mathbb{Z}$ one has $0 \in \operatorname{spec} A A^{*}$.

Proof. In view of periodicity, it is sufficient to show that the equation $A A^{*} \psi=0$ has non-trivial bounded solutions, $\psi \in \ell^{\infty}\left(\mathscr{V}_{1} ; \mathbb{C}^{2}\right)$.

Let us classify the nodes $\boldsymbol{\beta}_{m, n}$ and $\gamma_{m, n}$ as shown in figure 2. Consider all vector-valued functions on $\mathscr{V}_{1}$ vanishing at the white marked nodes. For such a function $\psi$, the condition $A^{*} \psi=0$ is of very simple form, because in the expression (21) only two of the six terms on the right-hand side are non-zero. Therefore, fixing the value of $\psi$ at a single black marked node one uniquely extends $\psi$ to a bounded solution of $A^{*} \psi=0$. The conditions $\omega \in \frac{\pi}{2}+\pi \mathbb{Z}$ and $k_{R} \in \pi \mathbb{Z}$ guarantee that this solution is well defined, i.e. that the phase factor along each cycle on the hexagonal lattice of black nodes is 1 . 
To summarize the previous considerations we note that the set spec $\widetilde{D}$ consists of the real $E$ satisfying $s\left(1 ; E+k_{R}^{2}\right)=0$. Proposition 7 reads as

Theorem 9. Let $\omega \in \frac{\pi}{2}+\pi \mathbb{Z}$ and $k_{R} \in \pi \mathbb{Z}$, then the spectrum of $L$ consists of the real numbers $E$ satisfying at least one of the following conditions:

$$
\begin{gathered}
\left(c\left(1 ; E+k_{R}^{2}\right)+\frac{\lambda}{6} s\left(1 ; E+k_{R}^{2}\right)\right) \cdot\left(s^{\prime}\left(1 ; E+k_{R}^{2}\right)+\frac{\mu}{3} s\left(1 ; E+k_{R}^{2}\right)\right)=\frac{1}{3}, \\
s^{\prime}\left(1 ; E+k_{R}^{2}\right)+\frac{\mu}{3} s\left(1 ; E+k_{R}^{2}\right)=0, \\
s\left(1 ; E+k_{R}^{2}\right)=0 .
\end{gathered}
$$

Each point of the spectrum is an infinitely degenerate eigenvalue.

Note that the eigenvalues (24a) are the most interesting ones, as they arise as the limit of the continuous spectrum. The Dirichlet eigenvalues (24c) are usually neglected in the physical works.

In the simplest case, when the scalar potential is zero and the couplings are trivial, i.e. $U=0, \lambda=\mu=0$, one has $s(x ; E)=\frac{1}{\sqrt{E}} \sin \sqrt{E} x, c(x ; E)=\cos \sqrt{E} x$, and Eq. 24a takes the form

$$
\cos ^{2} \sqrt{E+k_{R}^{2}} \in\left\{0, \frac{1}{3}, 1\right\}
$$

which was previously obtained in [54] for the case $k_{R}=0$.

We remark that the presence of the extreme localization is periodic with respect to the shifts $k_{R} \mapsto k_{R}+\pi$, but not the energy levels themselves, as the functions $s\left(\cdot ; E+k_{R}^{2}\right)$ etc. are not periodic with respect to the Rashba constant. It is worthwhile to note that the above results hold for any potential $U$ and any coupling constants $\lambda$ and $\mu$.

\subsection{Magneto-spin induced localization}

Another interesting situation appears at $\cos k_{R}=0$, i.e. at $k_{R} \in \frac{\pi}{2}+\pi \mathbb{Z}$. In this case one has

$$
A^{*} A=6-2\left(\begin{array}{cc}
\cos \left(\omega-\frac{\pi}{3}\right) \Delta & 0 \\
0 & \cos \left(\omega+\frac{\pi}{3}\right) \Delta
\end{array}\right) .
$$

For the values $\omega \in-\frac{\pi}{6}+\pi \mathbb{Z}$ the first component of $A^{*} A$ degenerates. In particular, any function of the form $(f, 0), f \in \ell^{2}\left(\mathbb{Z}^{2}\right)$ becomes an eigenfunction of $A^{*} A$. For $\omega \in \frac{\pi}{6}+\pi \mathbb{Z}$ the same holds for the functions $(0, f)$.

For further analysis we calculate the spectrum of $\Delta$. 
Lemma 10. For $\omega \in \pm \frac{\pi}{6}+\pi \mathbb{Z}$ the spectrum of $\Delta$ is absolutely continuous and covers the segments $[-2 \sqrt{3},-\sqrt{3}]$ and $[\sqrt{3}, 2 \sqrt{3}]$.

Proof. Consider the unitary transformation

$$
U: \ell^{2}\left(\mathbb{Z}^{2}\right) \ni(f(m, n)) \mapsto\left(\mathrm{e}^{3 \mathrm{i} \omega m n} f(m, n)\right) \in \ell^{2}\left(\mathbb{Z}^{2}\right) .
$$

Clearly, $U$ is unitary, and the operator $\hat{\Delta}:=U^{*} \Delta U$ has the form

$$
\begin{aligned}
\hat{\Delta} f(m, n)= & \mathrm{e}^{-6 \mathrm{i} \omega m} f(m, n+1)+\mathrm{e}^{6 \mathrm{i} \omega m} f(m, n-1)+f(m+1, n)+f(m-1, n) \\
& +\mathrm{e}^{-3 \mathrm{i} \omega(2 m-1)} f(m-1, n+1)+\mathrm{e}^{3 \mathrm{i} \omega(2 m+1)} f(m+1, n-1) .
\end{aligned}
$$

The operator obtained has the same spectrum as $\Delta$, but is periodic with respect to the shifts $n \mapsto n+1$ and can be studied using the Bloch analysis. Making the Bloch substitution $f(m, n)=\mathrm{e}^{\mathrm{i} n q} \xi_{m}$, where $q \in[0,2 \pi)$ is the quasimomentum, we observe that the spectrum of $\hat{\Delta}$ is the union of the spectra of operators $H(q)$ acting in $\ell^{2}(\mathbb{Z})$ and defined by

$$
\begin{aligned}
& (H(q) \xi)_{m}=2 \exp \left[-3 \mathrm{i} \omega\left(m-\frac{1}{2}\right)+\mathrm{i} \frac{q}{2}\right] \cos \left[3 \omega\left(m-\frac{1}{2}\right)-\frac{q}{2}\right] \xi_{m-1} \\
& +2 \cos [6 \omega m-q] \xi_{m}+2 \exp \left[3 \mathrm{i} \omega\left(m+\frac{1}{2}\right)-\mathrm{i} \frac{q}{2}\right] \cos \left[3 \omega\left(m+\frac{1}{2}\right)-\frac{q}{2}\right] \xi_{m+1} .
\end{aligned}
$$

The operators $H(q)$ are nothing but the Harper operators for the triangular lattice [18]. Note that for $\omega \in \pm \frac{\pi}{6}+\pi \mathbb{Z}$ all these operators are invariant under the shift $m \mapsto m+2$. Therefore, substituting into the equation $H(q) \xi=E \xi$ a vector $\xi$ satisfying $\xi_{m}=\mathrm{e}^{\mathrm{i} \theta} \xi_{m-2}$ for all $m$, where $\theta \in[0,2 \pi)$ is another quasimomentum, one arrives at a $2 \times 2$ linear system for the components $\xi_{0}$ and $\xi_{1}$,

$$
\begin{aligned}
\mathrm{e}^{\mathrm{i}(3 \omega / 2+q / 2)} \cos \left(\frac{3 \omega}{2}+\frac{q}{2}\right) \mathrm{e}^{-\mathrm{i} \theta} \xi_{1} & +\cos q \xi_{0} \\
& +\mathrm{e}^{\mathrm{i}(3 \omega / 2-q / 2)} \cos \left(\frac{3 \omega}{2}-\frac{q}{2}\right) \xi_{1}=\frac{E}{2} \xi_{0}, \\
\mathrm{e}^{-\mathrm{i}(3 \omega / 2-q / 2)} \cos \left(\frac{3 \omega}{2}-\frac{q}{2}\right) \xi_{0}-\cos q \xi_{1} & \\
& +\mathrm{e}^{-\mathrm{i}(3 \omega / 2+q / 2)} \cos \left(\frac{3 \omega}{2}+\frac{q}{2}\right) \mathrm{e}^{\mathrm{i} \theta} \xi_{0}
\end{aligned}
$$

The condition for the determinants to vanish takes the form

$$
\begin{aligned}
\frac{E^{2}}{4} & =1+\cos ^{2} q+\cos (q-\theta) \cos q \\
& =\frac{3}{4}+\left(\cos q+\frac{1}{2} \cos (q-\theta)\right)^{2}+\sin ^{2}(q-\theta) .
\end{aligned}
$$

Taking here all possible values of $q$ and $\theta$ we arrive at the conclusion. 
Lemma 10 means that for values $\omega$ in question, the spectrum of $A^{*} A$ has a continuous part, which is the union of the segments $[0,3]$ and $[9,12]$, and an infinitely degenerate eigenvalue 6 .

Therefore, we arrive, as in subsection 3.4 to a series of infinitely degenerate eigenvalues $E$ satisfying the same equation (24a) (i.e. the same eigenvalues as in the extreme localization case), which are isolated in the spectrum, but we have additionally bands of continuous spectrum given by

$$
\left(c\left(1 ; E+k_{R}^{2}\right)+\frac{\lambda}{6} s\left(1 ; E+k_{R}^{2}\right)\right) \cdot\left(s^{\prime}\left(1 ; E+k_{R}^{2}\right)+\frac{\mu}{3} s\left(1 ; E+k_{R}^{2}\right)\right) \in\left[0, \frac{1}{6}\right] \cup\left[\frac{1}{2}, \frac{2}{3}\right] .
$$

In particular, for the free case with zero coupling constants one has the following characterization for $E$ to be in the spectrum of $L$ :

$$
\cos ^{2} \sqrt{E+k_{R}^{2}} \in\left[0, \frac{1}{6}\right] \cup\left\{\frac{1}{3}\right\} \cup\left[\frac{1}{2}, \frac{2}{3}\right] \cup\{1\} .
$$

The localization effect described in this subsection seems to be not covered by the existing works, and it would be interesting to know whether it can be really observed. As for different values of the magnetic parameteres we have completely different eigensubspaces of $A^{*} A$, we conjecture that this localization mechanism can be used to control the spin polarization by the magnetic field, but this needs a further analysis.

\section{Appendix. Supersymmetric spectral analysis}

Here we prove the following proposition.

Proposition 11. Let $\mathscr{H}_{1}, \mathscr{H}_{2}$ are some Hilbert spaces, $A$ be a bounded linear operator from $\mathscr{H}_{1}$ to $\mathscr{H}_{2}$, and $m \in \mathbb{R}$. On $\mathscr{H}_{1} \oplus \mathscr{H}_{2}$ consider the operator

$$
L=\left(\begin{array}{cc}
m & A^{*} \\
A & -m
\end{array}\right)
$$

Then

$$
\operatorname{spec} L=-\sqrt{\operatorname{spec}\left(A A^{*}+m^{2}\right)} \cup \sqrt{\operatorname{spec}\left(A^{*} A+m^{2}\right)} .
$$

This proposition is formulated (without proof) in [43] and is nothing but an abstract version of proposition 2.5 in [49]; we give here a complete proof just for the sake of completeness.

Proof. First note that $\operatorname{spec} A A^{*} \backslash\{0\}=\operatorname{spec} A^{*} A \backslash\{0\}$ [20]. Clearly,

$$
L^{2}=\left(\begin{array}{cc}
A^{*} A+m^{2} & 0 \\
0 & A A^{*}+m^{2}
\end{array}\right) \text {. }
$$


Therefore, $\operatorname{spec} L^{2} \backslash\{ \pm m\}=\operatorname{spec}\left(A A^{*}+m^{2}\right) \backslash\left\{m^{2}\right\}$, and for any $\lambda \in \operatorname{spec} A A^{*} \backslash$ $\{0\} \equiv \operatorname{spec} A A^{*} \backslash\{0\}$ at least one of the numbers $-\sqrt{\lambda+m^{2}}, \sqrt{\lambda+m^{2}}$ lies in $\operatorname{spec} L$. Let us show that actually they both are in the spectrum of $L$.

Let $\lambda>0, \lambda \in \operatorname{spec} A^{*} A$, then there exist a sequence $\left(\phi_{n}\right), \phi_{n} \in \mathscr{H}_{1}$ such that $\left\|\phi_{n}\right\| \geq 1$ and $\lim \left(A^{*} A-\lambda\right) \phi_{n}=0$. Denote

$$
\psi_{n}:=\left[\lambda+\left(\sqrt{\lambda+m^{2}}-m\right)\left(\begin{array}{cc}
0 & A^{*} \\
A & 0
\end{array}\right)\right]\left(\begin{array}{c}
\phi_{n} \\
0
\end{array}\right)
$$

Clearly,

$$
\left(\begin{array}{c}
\phi_{n} \\
0
\end{array}\right) \perp\left(\begin{array}{cc}
0 & A^{*} \\
A & 0
\end{array}\right)\left(\begin{array}{c}
\phi_{n} \\
0
\end{array}\right)
$$

which implies

$$
\left\|\psi_{n}\right\| \geq \lambda\left\|\phi_{n}\right\| \geq \lambda
$$

By direct calculation,

$$
\left(L-\sqrt{\lambda+m^{2}}\right) \psi_{n}=\left(\sqrt{\lambda+m^{2}}-m\right)\left(\begin{array}{c}
\left(A^{*} A-\lambda\right) \phi_{n} \\
0
\end{array}\right) .
$$

Therefore, $\lim \left(L-\sqrt{\lambda+m^{2}}\right) \psi_{n}=0$. Together with (28) this implies $\sqrt{\lambda+m^{2}} \in$ spec $L$.

To show $-\sqrt{\lambda+m^{2}} \in \operatorname{spec} L$ one has to consider the functions

$$
\psi_{n}:=\left[\lambda-\left(\sqrt{\lambda+m^{2}}-m\right)\left(\begin{array}{cc}
0 & A^{*} \\
A & 0
\end{array}\right)\right]\left(\begin{array}{c}
0 \\
\phi_{n}
\end{array}\right),
$$

where $\left\|\phi_{n}\right\| \geq 1$ and $\lim \left(A A^{*}-\lambda\right) \phi_{n}=0$ and to repeat the above steps. To finish the proof of Eq. (25) it is necessary to study the points $\pm m$.

For $m=0$, Eq. (26) reads as $\operatorname{spec} L^{2}=\operatorname{spec} A A^{*} \cup \operatorname{spec} A^{*} A$, and the conditions $0 \in \operatorname{spec} L$ and $0 \in \operatorname{spec} A A^{*} \cup \operatorname{spec} A^{*} A$ are equivalent.

Assume $m \neq 0$ and $m \in \operatorname{spec} L$, then there exist sequences $\left(\phi_{n}\right) \in \mathscr{H}_{1},\left(\varphi_{n}\right) \in$ $\mathscr{H}_{2}$ with

$$
\left\|\phi_{n}\right\|+\left\|\varphi_{n}\right\| \geq 1
$$

and

$$
\lim (L-m)\left(\begin{array}{c}
\phi_{n} \\
\varphi_{n}
\end{array}\right) \equiv \lim \left(\begin{array}{c}
A^{*} \varphi_{n} \\
A \phi_{n}-2 m \varphi_{n}
\end{array}\right)=0 .
$$

Clearly, this implies $\lim A^{*} A \phi_{n}=0$. Assume that $\lim \phi_{n}=0$, then (30) shows $\lim \varphi_{n}=0$ which contradicts (29). Therefore, there exists a subsequence $\left(\phi_{n}^{\prime}\right)$ of $\left(\phi_{n}\right)$ such that $\left\|\phi_{n}^{\prime}\right\| \geq \varepsilon$ for some $\varepsilon>0$. Together with $\lim A^{*} A \phi_{n}^{\prime}=0$ this implies $0 \in \operatorname{spec} A^{*} A$. 
Assume now $0 \in \operatorname{spec} A^{*} A$, then there is a sequence $\left(\phi_{n}\right) \in \mathscr{H}_{1}$ with $\left\|\phi_{n}\right\| \geq 1$ and $\lim \left\langle A^{*} A \phi_{n}, \phi_{n}\right\rangle \equiv \lim \left\|A \phi_{n}\right\|=0$. Then

$$
\lim (L-m)\left(\begin{array}{c}
\phi_{n} \\
0
\end{array}\right)=\lim \left(\begin{array}{c}
0 \\
A \phi_{n}
\end{array}\right)=0
$$

from which $m \in \operatorname{spec} L$.

The relationship between the conditions $-m \in L$ and $0 \in \operatorname{spec} A A^{*}$ can be proved in a completely similar way.

It may be useful to have an alternative formulation of proposition 11

Corollary 12. There holds

$$
\begin{aligned}
\operatorname{spec} L \backslash\{-m, m\} & =-\sqrt{\operatorname{spec}\left(A A^{*}+m^{2}\right)} \cup \sqrt{\operatorname{spec}\left(A A^{*}+m^{2}\right)} \backslash\{-m, m\} \\
& \equiv-\sqrt{\operatorname{spec}\left(A^{*} A+m^{2}\right)} \cup \sqrt{\operatorname{spec}\left(A^{*} A+m^{2}\right)} \backslash\{-m, m\}
\end{aligned}
$$

Furthermore, for $m \neq 0$ one has: $m \in \operatorname{spec} L$ iff $0 \in \operatorname{spec} A^{*} A,-m \in \operatorname{spec} L$ iff $0 \in \operatorname{spec} A A^{*}$, and for $m=0$ there holds $0 \in \operatorname{spec} L$ iff $0 \in \operatorname{spec} A^{*} A \cup \operatorname{spec} A A^{*}$.

\section{Acknowledgments}

Numerous discussions with Vladimir Geyler and Denis Bulaev are gratefully acknowledged.

The research was supported by the Deutsche Forschungsgemeinschaft (fellowship PA 1555/1-1) and the German-New Zealand cooperation of BMBF (project no. NZL 05/001 of the International Bureau at the German Aerospace Center) and ISAT (grant no. 9144/360402 of the Royal Society of New Zealand). A large part of the work was done during the stay at the Department of Mathematics of the University of Auckland in March 2006. The author thanks Boris Pavlov for valuable interaction and the warm hospitality during the visit.

\section{References}

[1] Abilio, C. C., Butaud, P., Fournier, Th., Pannetier, B., Vidal, J., Tedesco, S., Dalzotto, B.: Magnetic field induced localization in a two-dimensional superconducting wire network, Phys. Rev. Lett. 83 (1999) 5102-5105.

[2] Aeppli, G., Chandra, P.: Seeking a simple complex system, Science 275 (1997) 177-178. 
[3] Akkermans, E., Comtet, A., Desbois, J., Montambaux, G., Texier, C.: Spectral determinant on quantum graphs, Ann. Phys. (New York) 284 (2000) $10-51$.

[4] Alexander, S.: Superconductivity of networks. A percolation approach to the effects of disorder, Phys. Rev. B 27 (1983) 1541-1557.

[5] von Below, J.: A characteristic equation associated to an eigenvalue problem on $c^{2}$-networks, Linear Algebra Appl. 71 (1985) 309-325.

[6] von Below, J.: Sturm-Liouville eigenvalue problems on networks, Math. Meth. Appl. Sci. 10 (1988) 383-395.

[7] Bercioux, D., Governale, M., Cataudella, V., Ramaglia, V. M.: Rashbaeffect-induced localization in quantum networks, Phys. Rev. Lett. 93 (2004) 056802.

[8] Bercioux, D., Governale, M., Cataudella, V., Ramaglia, V. M.: Rashba effect in quantum networks, Phys. Rev. B 72 (2005) 075305.

[9] Birman, M. Sh., Suslina, T. A.: A periodic magnetic Hamiltonian with a variable metric. The problem of absolute continuity, St. Petersburg Math. J. 11 (2000) 203-232.

[10] Bolte, J., Harrison, J.: Spectral statistics for the Dirac operator on graphs, J. Phys. A: Math. Gen. 36 (2003) 2747-2769.

[11] Bolte, J., Harrison, J.: The spin contribution to the form factor of quantum graphs, J. Phys. A: Math. Gen. 36 (2003) L433-L440.

[12] Brüning, J., Geyler, V., Pankrashkin, K.: Cantor and band spectra for periodic quantum graphs with magnetic fields, Commun. Math. Phys. (to appear), Preprint math-ph/0511057.

[13] Bulla, W., Trenkler, T.: The free Dirac operator on compact and noncompact graphs, J. Math. Phys. 31 (1990) 1157-1163.

[14] Bychkov, Yu. A., Rashba, E. I.: Properties of a 2D electron gas with lifted spectral degeneracy, Sov. Phys. JETP Lett. 39 (1984) 78-80.

[15] Castro, J. I., López, A.: The de Gennes-Alexander theory of superconducting micronetworks, In: Berger, J., Rubinstein, J. (Eds.): Connectivity and Superconductivity (Lecture Notes Phys. Monographs, vol. 62, Springer, Berlin, 2000) 23-62 . 
[16] Cattaneo, C.: The spectrum of the continuous Laplacian on a graph. Monatsh. Math. 124 (1997) 215-235.

[17] Chaplik, A. V., Magarill, L. I.,: Bound states in a two-dimensional short range potential induced by the spin-orbit interaction, Phys. Rev. Lett. 96 (2006) 126402.

[18] Claro, F. H., Wannier, G. H.: Magnetic subband structure of electrons in hexagonal lattices, Phys. Rev. B 19 (1979) 6068-6074.

[19] Debald, S., Kramer, B.: Rashba effect and magnetic field in semiconductor quantum wires, Phys. Rev. B 71 (2005) 115322.

[20] Deift, P.: Applications of a commutation formula, Duke Math. J. 45 (1978) 267-310.

[21] Derkach, V. A., Malamud, M. M.: Generalized resolvents and the boundary value problems for Hermitian operators with gaps, J. Funct. Anal. 95 (1991) $1-95$.

[22] Desbois, J.: Spectral determinant on graphs with generalized boundary conditions, Eur. Phys. J. B 24 (2001) 261-266.

[23] Exner, P.: A duality between Schrödinger operators on graphs and certain Jacobi matrices, Ann. Inst. Henri Poincaré Phys. Théor. 66 (1997) 359-371.

[24] Exner, P.: Lattice Kronig-Penney models, Phys. Rev. Lett. 74 (1995) 35033506.

[25] de Gennes, P.-G.: Diamagnétisme de grains supraconducteurs près d'un seuil de percolation, C. R. Acad. Sci. Paris Sér. II 292 (1981) 9-12.

[26] Geyler, V. A., Margulis, V. A.: Anderson localization in the nondiscrete Maryland model, Theor. Math. Phys. 70 (1987) 133-140.

[27] Gnutzmann, S., Altland, A.: Spectral correlation of individual quantum graphs, Phys. Rev. E 72 (2005) 056215.

[28] Kostrykin, V., Schrader, R.: Kirchhoff's rule for quantum wires, J. Phys. A: Math. Gen. 32 (1999) 595-630.

[29] Kostrykin, V., Schrader, R.: Quantum wires with magnetic fluxes, Commun. Math. Phys. 237 (2003) 161-179.

[30] Kottos, T., Smilansky, U.: Periodic orbit theory and spectral statistics for quantum graphs, Ann. Phys. (New York) 274 (1999) 76-124. 
[31] Kuchment, P.: Graph models for waves in thin structures, Waves Random Media 12 (2002) R1-R24.

[32] Kuchment, P.: Quantum graphs I. Some basic structures, Waves Random Media 14 (2004) S107-S128.

[33] Kuchment, P.: Quantum graphs II. Some spectral properties of quantum and combinatorial graphs, J. Phys. A: Math. Gen. 38 (2005) 4887-4900.

[34] Magarill, L. I., Chaplik, A. V.: Spin-dependent electron localization in crystals, JETP Lett. 81 (2005) 162-166.

[35] Mikhailova, A., Pavlov, B., Prokhorov, L.: Modelling of quantum networks, Preprint math-ph/0312038

[36] Mills, R. G. J., Montroll, E. W.: Quantum theory on a network II. A solvable model which may have several bound states per node point, J. Math. Phys. 11 (1970) 2525-2538.

[37] Montroll, E. W.: Quantum theory on a network I. A solvable model whose eigenfunctions are elementary functions, J. Math. Phys. 11 (1970) 635-648.

[38] Moulopoulos, K., Constantinou, M.: Magnetic-field-induced localization in networks with the $T_{3}$ geometry, Phys. Lett. A 302 (2002) 39-47.

[39] Naud, C.: Transport quantique dans des nanostructures, Ann. Phys. (Paris) 27 no. 5 (2002) 1-140.

[40] Naud, C., Faini, G., Mailly, D.: Aharonov-Bohm cages in 2D normal metal networks, Phys. Rev. Lett. 81 (1998) 5888-5891.

[41] Naud, C., Faini, G., Mailly, D., Vidal, J., Douçot, B., Montambaux, G., Wieck, A., Reuter, D.: Aharonov-Bohm cages in the GaAlAs/GaAs system, Physica E 12 (2002) 190-196.

[42] Nicaise, S.: Spectre des réseaux topologiques finis, Bull. Sci. Math. 111 (1987) 401-413.

[43] Ogurisu, O.: Supersymmetric analysis of the spectral theory on infinite graphs, Preprint 02-242 on http: / / www.ma.utexas.edu/mp_arc/

[44] Pankrashkin, K.: Spectra of Schrödinger operators on equilateral quantum graphs, Lett. Math. Phys. 77 (2006) 139-154.

[45] Pankrashkin, K.: Spectral duality for discrete and continuous Schrödinger operators on graphs and hypergraphs, in preparation. 
[46] Pavlov, B. S.: The theory of extensions and explicitly solvable models, Russian Math. Surveys 42 (1987) 127-168.

[47] Rashba, E. I.: Properties of semiconductors with an extremum loop. 1. Cyclotron and combinational resonance in a magnetic field perpendicular to the plane of the loop, Sov. Phys. Solid State 2 (1960) 1109-1122.

[48] Roth, J.-P.: Spectre du laplacien sur un graphe, C. R. Acad. Sci. Paris 296 (1983) 783-795.

[49] Shigekawa, I.: Spectral properties of Schrödinger operators with magnetic fields for a spin $\frac{1}{2}$ particle, J. Funct. Anal. 101 (1991) 255-285.

[50] Sobolev, A. V.: Absolute continuity of the periodic magnetic Schrödinger operator, Invent. Math. 137 (1999) 85-112.

[51] Sutherland, B.: Localization of electronic wave functions due to local topology, Phys. Rev. B 34 (1986) 5208-5211.

[52] Vidal, J., Butaud, P., Douçot, B., Mosseri, R.: Disorder and interactions in Aharonov-Bohm cages, Phys. Rev. B 64 (2001) 155306.

[53] Vidal, J., Douçot, B., Mosseri, R., Butaud, P.: Interaction induced delocalization for two particles in a periodic potential, Phys. Rev. Lett. 85 (2000) 3906-3909.

[54] Vidal, J., Mosseri, R., Douçot, B.: Aharonov-Bohm cages in two-dimensional structures, Phys. Rev. Lett. 86 (2001) 5104-5107. 